\title{
Glycine decarboxylase deficiency-induced motor dysfunction in zebrafish is rescued by counterbalancing glycine synaptic level
}

\author{
Raphaëlle Riché, ${ }^{1}$ Meijiang Liao, ${ }^{1}$ Izabella A. Pena, ${ }^{2}$ Kit-Yi Leung, ${ }^{3}$ Nathalie Lepage, ${ }^{2}$ \\ Nicolas D.E. Greene, ${ }^{3}$ Kyriakie Sarafoglou, ${ }^{4}$ Lisa A. Schimmenti, ${ }^{5,6,7}$ Pierre Drapeau, ${ }^{1,8}$ \\ and Éric Samarut ${ }^{1,8}$
}

'Research Center of the University of Montreal Hospital Center (CRCHUM), Department of Neurosciences, Université de Montréal, Montreal, Quebec, Canada. ²Children's Hospital of Eastern Ontario Research Institute and Department of Pediatrics, Faculty of Medicine, University of Ottawa, Ontario, Canada. ${ }^{3}$ Developmental Biology and Cancer Programme, UCL Great Ormond Street Institute of Child Health, University College London, London, United Kingdom. ${ }^{4}$ Division of Pediatric Endocrinology, Department of Pediatrics, University of Minnesota, Minneapolis, Minnesota, USA. ${ }^{5}$ Department of Otorhinolaryngology, ${ }^{6}$ Department of Pediatrics, and ${ }^{7}$ Department of Clinical Genomics, Mayo Clinic College of Medicine, Rochester, Minnesota, USA. ${ }^{8}$ DanioDesign Inc., Montréal, Quebec, Canada.

Glycine encephalopathy (GE), or nonketotic hyperglycinemia (NKH), is a rare recessive genetic disease caused by defective glycine cleavage and characterized by increased accumulation of glycine in all tissues. Here, based on new case reports of CLDC loss-of-function mutations in GE patients, we aimed to generate a zebrafish model of severe GE in order to unravel the molecular mechanism of the disease. Using CRISPR/Cas9, we knocked out the gldc gene and showed that gldc $^{-/-}$fish recapitulate GE on a molecular level and present a motor phenotype reminiscent of severe GE symptoms. The molecular characterization of $\mathrm{gldc}^{-/-}$mutants showed a broad metabolic disturbance affecting amino acids and neurotransmitters other than glycine, with lactic acidosis at stages preceding death. Although a transient imbalance was found in cell proliferation in the brain of $\mathrm{gldc}^{-/-}$zebrafish, the main brain networks were not affected, thus suggesting that CE pathogenicity is mainly due to metabolic defects. We confirmed that the gldc $^{-/-}$hypotonic phenotype is due to NMDA and glycine receptor overactivation, and demonstrated that gldc $^{-/-}$larvae depict exacerbated hyperglycinemia at these synapses. Remarkably, we were able to rescue the motor dysfunction of $\mathrm{gldc}^{-/-}$larvae by counterbalancing pharmacologically or genetically the level of glycine at the synapse.

Conflict of interest: The authors have declared that no conflict of interest exists.

License: Copyright 2018, American Society for Clinical Investigation.

Submitted: August 31, 2018 Accepted: September 19, 2018 Published: November 2, 2018

\section{Reference information:} JCI Insight. 2018;3(21):e124642. https://doi.org/10.1172/jici. insight.124642.

\section{Introduction}

Glycine encephalopathy (GE), also known as nonketotic hyperglycinemia (NKH; OMIM 605899), is a rare genetic defect in glycine metabolism characterized by considerable accumulation of glycine in all tissues, especially the central nervous system (1-4). GE has an autosomal recessive inheritance pattern and is classically caused by mutations in proteins of the glycine cleavage system (GCS) composed of a glycine decarboxylase (GLDC), an aminomethyltransferase (AMT), a hydrogen carrier protein (GCSH), and a dihydrolipoamide dehydrogenase (DLD) $(4,5)$. All 4 subunits are encoded by distinct genes, and GLDC is mutated in $72 \%$ of the GE cases (2).

The symptoms of GE are commonly first observed during the neonatal period and are very heterogeneous among patients, depending on the pathogenicity of the mutation $(5,6)$. In severe GE, neonates present severe hypotonia, myoclonic jerks, lethargy, and apnea due to respiratory depression, which often causes death within the first week of life. Patients with severe GE surviving the neonatal period make no developmental progress and show spasticity, intractable seizures, and hypotonia. Individuals with attenuated GE often survive the neonatal period but show treatable seizures, spasticity, chorea, and variable developmental delay that can lead to intellectual disability $(5,6)$.

The treatments available for GE patients are primarily used to alleviate symptoms but do not resolve the underlying metabolic defects. Indeed, dextromethorphan, an NMDA receptor antagonist, is used to diminish 
seizures, and sodium benzoate helps reduce glycine levels by elimination through the urine. Unfortunately, even when combined, these treatments fail to improve the outcome for many GE patients (6). However, although the clinical research done on human patients has helped identify the genetic causes of the disease and characterize the spectrum of symptoms, it has not elucidated the molecular basis of GE.

A few research efforts have been made to model the disease. Mice with a loss-of-function gene trap allele and those with a dominant-negative gldc mutation, showing features of GE, such as early lethality, increased glycine, and hydrocephalus, were generated $(7,8)$. A zebrafish model was described in which hyperglycinemia is restricted to the brain, but it cannot be used to study the classical form of the disease (9). These models helped elucidate aspects of GE, but did not focus on characterizing the pathogenic mechanisms underlying the disease. This highlights the necessity of generating an accurate and reliable animal model of GE that is more amenable to metabolic analyses and high-throughput drug screens.

Here we report 2 new cases of GE patients carrying loss-of-function mutations in one or both GLDC alleles. In light of this identification, we generated a zebrafish model of GE ( $\left.\operatorname{lldc}^{--}\right)$, with a high level of glycine and displaying motor abnormalities and premature death, thus recapitulating the fatal outcome of severe forms of GE. Next, we performed unbiased high-throughput proteomics and transcriptomics analysis and showed that gldc loss of function induces broad metabolic defects. We also confirmed synaptic glycine signaling abnormalities and remarkably were able to rescue the hypotonic phenotype of $\mathrm{gldc}^{-1-}$ larvae by counterbalancing the hyperglycinemia at the synapse.

\section{Results}

Two case reports of GE associated with monoallelic or biallelic loss-of-function mutations in GLDC. The first patient we examined was a deceased female infant born at the gestational age of 39 weeks. Pregnancy was uncomplicated, and she was delivered vaginally, with Apgar scores of 8 at 1 minute and 9 at 5 minutes. She was discharged from the nursery at 2 days of life. At 4 days of life, she became lethargic, with poor feeding, and was admitted again. Her physical examination was significant for respiratory failure requiring intubation, diffuse hypotonia, absent deep tendon reflexes, withdrawal to painful stimuli, rhythmic hiccupping, and myoclonic movements of the right upper extremity. MRI on day 4 of life revealed a small but completely formed corpus callosum with a mild delay in myelination and a slightly lower volume of cerebral white matter than in healthy patient brains. EEG revealed a burst suppression pattern. Her seizures were treated with phenobarbital and Ativan, but were relatively refractory to medication. Additional testing revealed an elevated plasma glycine level of $125 \mu \mathrm{mol} / \mathrm{dl}$ (normal range, $0-57$ ) and elevated cerebrospinal fluid (CSF) glycine of $33.8 \mu \mathrm{mol} / \mathrm{dl}$ (normal range, $0.2-2.0$ ). The CSF/plasma glycine ratio of 0.27 (pathognomonic ratio $>0.08$ ) was diagnostic of GE (or NKH). Given the grave prognosis of this disease, the infant's family decided to withdraw life-sustaining treatment on day 10 of life. Newborn screening was negative and chromosomal studies were pending at the time of death. Molecular testing revealed 2 variants in the GLCD gene: c.1153 C>T (p.Q385X) and c.941 ins16nt fs (Table 1 and Figure 1).

The second patient was a 50-year-old man who presented initially to adult neurology for a movement disorder. He was nonverbal, had limited facial expression, drooled, and had a constant tremor. He was unable to walk unassisted and required caretakers to assist with personal tasks such as feeding and toileting. He had a history of myoclonic jerks and was treated with valproic acid. Clinical evaluation identified a mild elevation of glycine. Genetic testing identified2 variants in GLCD: c.1054delA(p. Thr352fster65) and c.1705C>T(p.Ala569Thr) (NM00170.2, Figure 1 and Table 1), and paternal testing supported that the variants were in trans. Although the first allele carrying a nonsense mutation is pathogenic, the second one with a missense mutation has been recently reported as nonpathogenic, with residual enzymatic activity of about $70 \%$ (10). After the diagnosis, valproic acid was promptly discontinued, and the patient was started on dextromethoraphan and sodium benzoate. Glycine levels fell into the normal range, and the patient had improved mobility and resolution of constant tremors, stopped drooling, and was able to feed himself. However, his general quality of life was still severely impaired, since he continued to need daily assistance.

These 2 cases demonstrate the clinical heterogeneity of GE and are consistent with a previous study linking the severity of the disease with the residual GLDC enzymatic activity of different missense mutations (11).

Zebrafish gldc is expressed in the CNS, and its loss of function induces glycine accumulation. We started by examining the $g l d c$ sequence and its expression in zebrafish embryos. In contrast to the common gene duplication 
Table 1. GE patient case reports

\begin{tabular}{|c|c|c|}
\hline & Patient 1 & Patient 2 \\
\hline Onset & Newborn (day 4) & Adult (50 years old) \\
\hline Gene & $G L D C$ & $G L D C$ \\
\hline Allele 2 & c. 941 ins16nt fs & c.1705C>T (p.A569T) \\
\hline First plasma glycine level & $125 \mu \mathrm{mol} / \mathrm{dl}$ (normal range, $0-57$ ) & $75 \mu \mathrm{mol} / \mathrm{dl}(13-50)$ \\
\hline CSF glycine level & $33.8 \mu \mathrm{mol} / \mathrm{dl}$ (normal range, $0.2-2.0$ ) & NA \\
\hline
\end{tabular}

in teleost fish, a single copy of the gldc gene can be found in the zebrafish genome (ENSDAR00000035120), and it shows more than $60 \%$ overall amino acid identity with the human protein. We also assessed the expression pattern of gldc during zebrafish development by in situ hybridization from 8 hours after fertilization (hpf) to $42 \mathrm{hpf}$. Gldc was broadly expressed in the nervous system until $32 \mathrm{hpf}$, and at $42 \mathrm{hpf}$ its expression was in specific brain regions (Figure 2A). Interestingly, we observed that the expression became restricted to the midbrain/hindbrain boundary and the commissural regions of the brain from 3 days after fertilization (dpf). From $4 \mathrm{dpf}$, gldc transcript started to be strongly expressed in the liver, the intestine, and the gut. This expression persisted at 5 and $6 \mathrm{dpf}$ with only weak expression in the brain.

The high degree of identity between human and zebrafish GLDC and its strong expression in the embryonic CNS suggest robust evolutionary conservation of GLDC function, thus supporting the pertinence of $g l d c-\mathrm{KO}$ zebrafish as a model for the human disease.

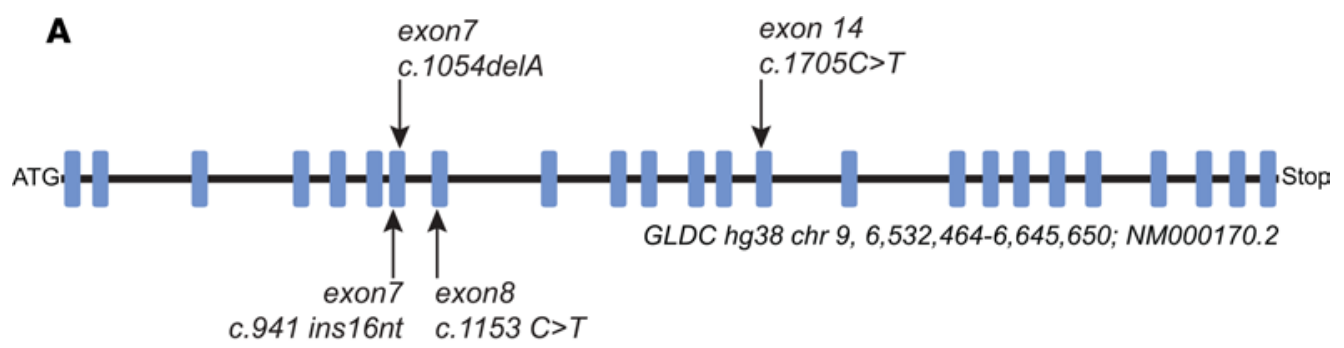

B

wildtype GLDC N-ter -11020
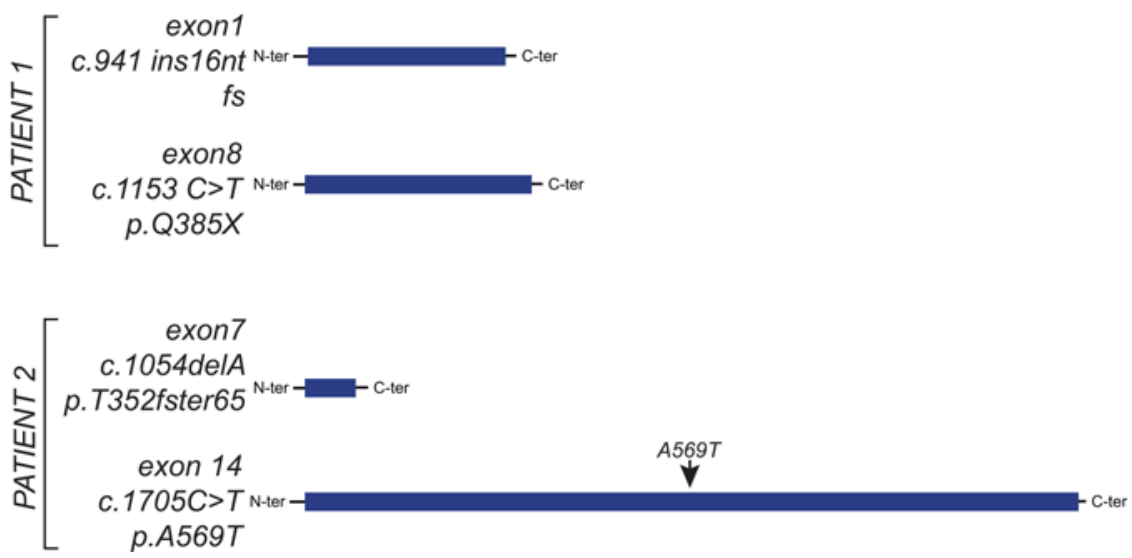

Figure 1. CLDC mutations associated with lethal or severe glycine encephalopathy. (A) The genetic position of each mutation is indicated on the scheme depicting the 25 exons of the GLDC gene. (B) Consequences of the mutations on the expected GLDC protein size. 
A<smiles>COc1ccccc1</smiles>

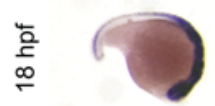

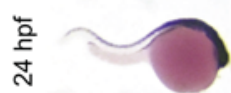

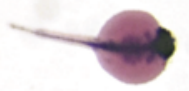

芩

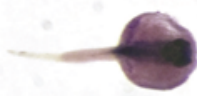

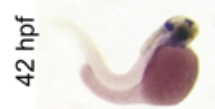

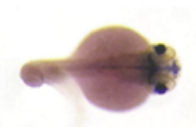

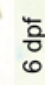

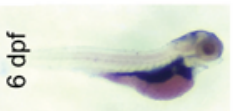

Figure 2. Zebrafish gldc is expressed in the CNS and its loss of function induces glycine accumulation. (A) In situ hybridization of gldc transcript at 8, 18, 24, 32, and 42 hours after fertilization (hpf) showing the expression in the brain and spinal cord as well as in the intestine at later stages. dpf, days after fertilization. (B) CRISPR/Cas9 mutagenesis targeting the 4th exon of gldc led to substitution of 2 nucleotides and deletion of 5 nucleotides. These DNA mutations in $g / d c^{-/-}$fish induce a melting curve shift compared with gldc $\mathrm{C}^{+/+}$that allows for rapid genotyping. (C) Scheme of several nonsense mutations identified in patients leading to insertion of stop codons in the glycine cleavage domain of the human GLDC protein. Red indicates the mutant variant of the glycine encephalopathy patient described here; and the location of the CRISPR mutation in the zebrafish CLDC protein. ter, terminal. (D) RT-qPCR analysis of gldc mRNA levels reveals a significant decrease in $\mathrm{gldc}^{-/+}$and gldc ${ }^{-1-}$ compared with gld $c^{+/+}$fish, at $7 \mathrm{dpf}$ (ANOVA, ${ }^{* *} P<$ $0.0004 ;{ }^{* *} P<0.005, n>10, N=3$ ). LC-MS dosage of glycine levels in whole $7 \mathrm{dpf}$ larvae reveals a significant increase in

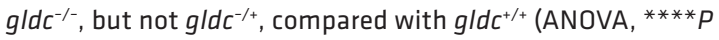
$<0.0001, n=3, n=3$ ). Boxes and whiskers represent minimum and maximum values, and a line shows the median value. Each dot corresponds to an individual experiment $(N)$ with at least 5 fish $(n>5)$.
D

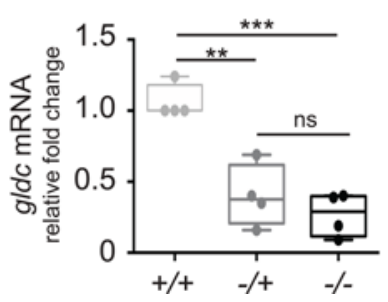

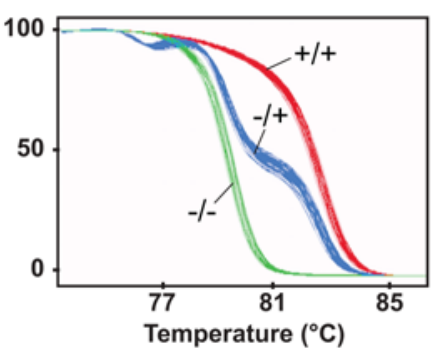

Temperature $\left({ }^{\circ} \mathrm{C}\right)$

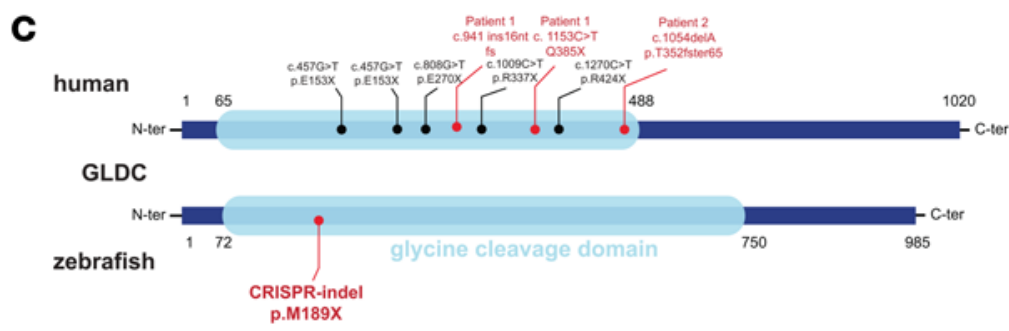

E

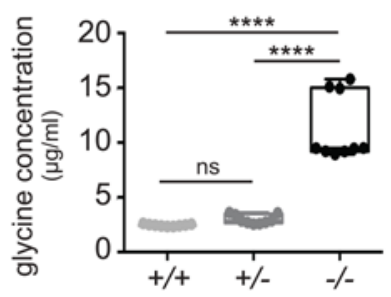

In designing guide RNAs (gRNAs) for CRISPR/Cas9-mediated mutagenesis, we targeted the beginning of the glycine cleavage domain, with the aim of reproducing a pathogenic loss-of-function mutation. We selected a founder transmitting a 5-nucleotide deletion and 3 nucleotide mismatches early in the glycine cleavage domain, creating a stop codon at the 189th amino acid (Figure 2, B and C). Using high-resolution melting (HRM) analysis (12), we could easily identify all 3 genotypes $(+/+,-/+,-/-)$ (Figure $2 \mathrm{~B}$ ). We confirmed gldc KO by real-time quantitative PCR (RT-qPCR) and showed that $g l d c^{-1-}$ embryos have reduced levels of gldc expression compared with their siblings, as seen in patients (13). More importantly, liquid chromatography-mass spectrometry (LC-MS) analysis of glycine levels in whole $7 \mathrm{dpf}$ larvae supported the loss-of-function nature of the mutation, since $\mathrm{gldc}^{-1-}$ larvae displayed 4 times more accumulated glycine than their siblings (Figure 2D). Altogether, these results show that our newly generated $g l d c-\mathrm{KO}$ line recapitulates the main molecular hallmarks of GE. 
A

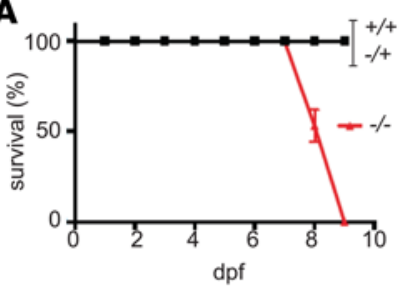

C

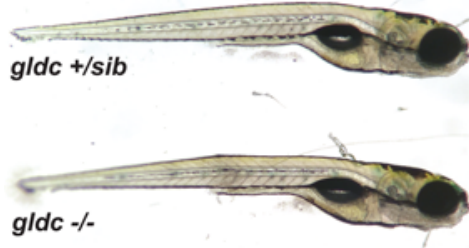

B

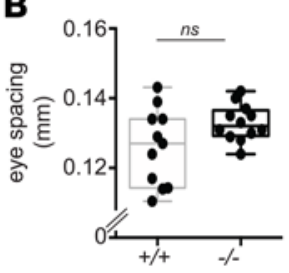

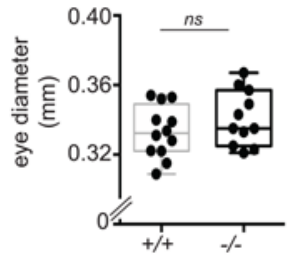

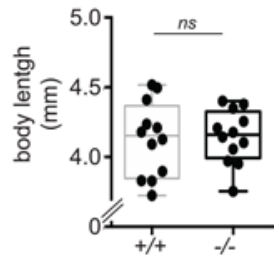

D

gldc $+/+$
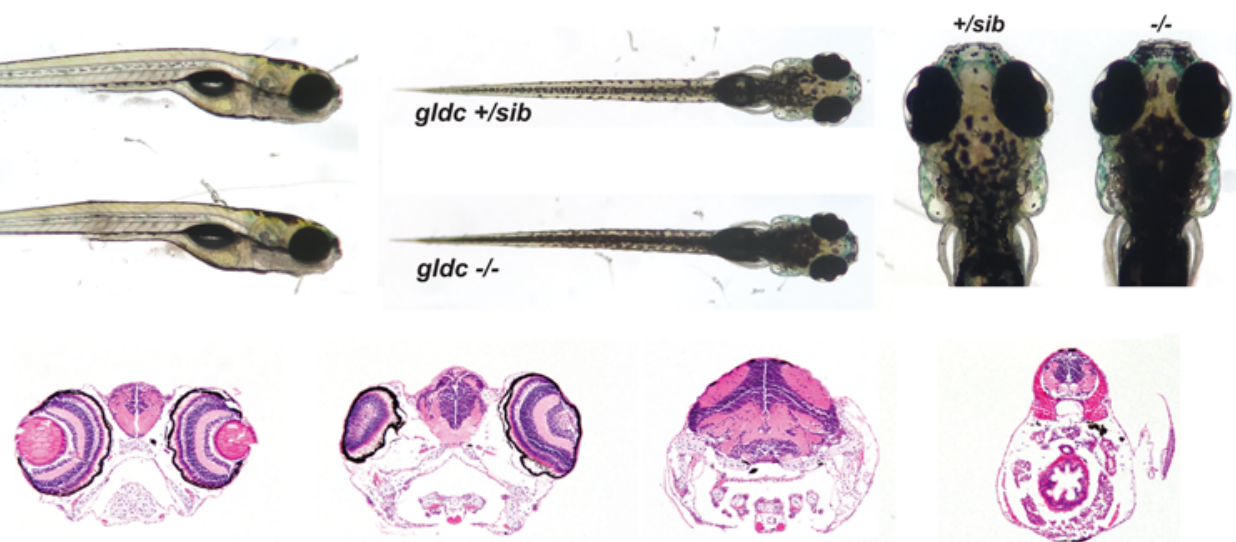

gldc $-/-$
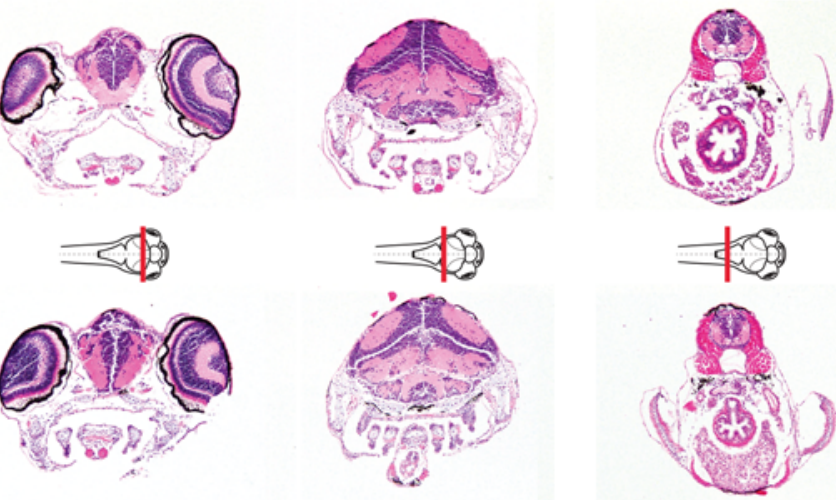

E

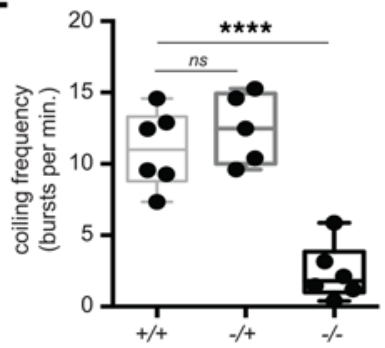

$\mathbf{F}$

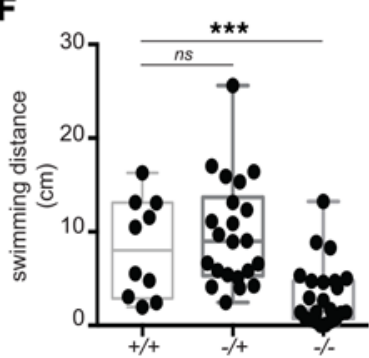

G
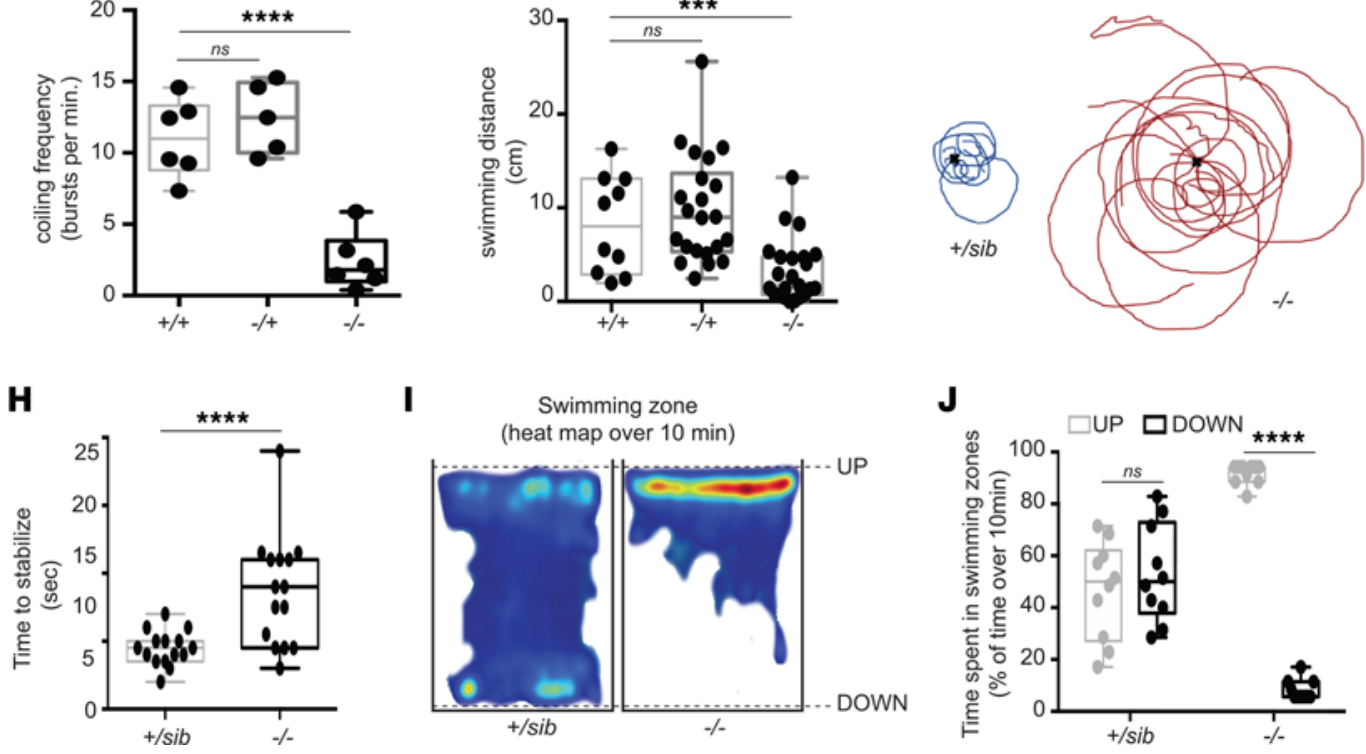

Figure 3. GIdc/- larvae die prematurely and show disease-reminiscent motor phenotypes. (A) Gld ${ }^{-/-}$larvae die prematurely between 7 and 9 days after fertilization (dpf). (B) The general morphology of the fish is not affected, as shown by the quantification of the eye spacing distance, eye diameter, and body length of $\mathrm{gldc}^{-/-}$compared with $\mathrm{gldc}^{+/+}$larvae. (C) Although there is no obvious morphological defect in $\mathrm{gldc}^{-/-}$at $7 \mathrm{dpf}$, $\mathrm{g} / \mathrm{dc} \mathrm{c}^{-/-}$larvae are distinguishable from their siblings because of their hyperpigmentation. (D) H\&E staining performed on transverse sections from $7 \mathrm{dpf} g / \mathrm{dc}^{+/+}$ and $\mathrm{gldc}^{-/-}$larvae shows no obvious difference in brain morphology. (E) Analysis of the frequency of spontaneous coiling of the tail at 21 hours after fertilization, the earliest motor phenotype in zebrafish embryo, reveals a decrease in this behavior in $\mathrm{gldc}^{-{ }^{--}}$compared with $\mathrm{gldc^{+/+ }}$ larvae (ANOVA $\left.{ }^{* * *} P=0.0002\right)$. (F) Analysis of the total distance swimming distance over 1 hour at 7 days reveals a significant decrease in $\mathrm{gldc}^{-1-}$ compared with gld $c^{+/+}$(ANOVA ${ }^{* *} P=0.0077$ ). (G) Tracking of the position of $g / d c^{+/+}$and $g / d c^{-/-} 7$-dpf larvae from the center point after application of a water current

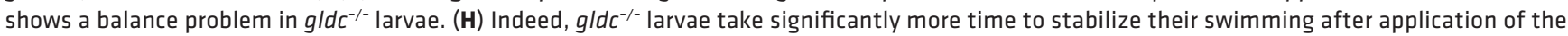
water current compared with $\mathrm{gldc^{+/+ }}\left(t\right.$ test, $\left.{ }^{* * * *} P=0.0008\right)$. (I) Heatmap tracking of the swimming zone of 7 -dpf larvae over 10 minutes reveals that 
gld $^{-/-}$larvae swim only in the upper part of water, whereas gldc $c^{+/+}$swim in both the upper and lower sections of the water. (J) Quantification of the time spent in the upper versus lower swimming zone of the water reveals that gld ${ }^{-/-}$larvae spend significantly more time in the upper swimming zone compared with gld $c^{+/}$(ANOVA, $\left.{ }^{* * *} P<0.0001\right)$. Boxes and whiskers represent minimum and maximum values, and a line shows the median value. Each dot corresponds to an individual experiment $(N)$ with at least 5 fish $(n>5)$. "+/sib" indicates $g / d c^{+/+}$and $g l d c^{-/+}$siblings.

gldc ${ }^{-1}$ larvae die prematurely and show GE-reminiscent motor phenotypes. While most $\mathrm{gldc}^{+/+}$and gldc $^{-/+}$larvae survived for the first $10 \mathrm{dpf}$, all $\mathrm{gldc}^{-1-}$ larvae died between 7 and $9 \mathrm{dpf}$ (Figure 3A). After measuring body length, eye size, and inter-eye distance of $\mathrm{gldc}^{-1}$ larvae compared with their siblings, we did not notice any morphological abnormalities in the mutant larvae (Figure 3B). However, hyperpigmentation was evident in $\mathrm{gldc}^{-1}$ larvae from $6 \mathrm{dpf}$, which allowed them to be easily distinguished from their siblings (Figure 3C). This hyperpigmentation phenotype segregated with the mutant alleles of gldc through multiple generations of fish, thus suggesting that, although unexpected, it was specific to gldc loss of function. Although no major morphological differences were observed, we analyzed transverse sections of different regions of the larval brain (forebrain, midbrain, hindbrain, and anterior spinal cord) and did not observe any obvious morphological differences between $\mathrm{gldc}^{+/+}$and $\mathrm{gldc}^{-/-}$fish at $7 \mathrm{dpf}$ (Figure 3D).

Since GE patients show early motor symptoms such as hypotonia and lethargy, we analyzed the larval motor function of the gldc mutants. Spontaneous coiling is the first motor output in zebrafish embryos, occurring between 17 and $21 \mathrm{hpf}$ and consisting of frequent unevoked movements of the tail in the chorion (14). Analysis of this motor behavior indicated a decrease in coiling frequency of gldc $^{-1-}$ embryos compared with their siblings $(1.46 \pm 0.31, n=14 ; 12.81 \pm 2.73, n=11$; Figure $3 \mathrm{E})$. At the larval stage, we assayed the swimming activity of gldc mutants by recording the total swimming distance over 1 hour, and we noticed a reduction in swimming activity in $\mathrm{gldc}^{-/}$larvae at $7 \mathrm{dpf}(650.6 \pm 138.4 \mathrm{~mm}, n=23 ; 1,646 \pm 332.1 \mathrm{~mm}, n=10$; Figure 3F). Of note is that this reduced swimming behavior started at $4 \mathrm{dpf}$ and was increasingly evident with age.

Hypotonia and lethargy are respectively defined as a state of low muscle tone and lack of vigor. Thus we tracked the path swum by $\mathrm{gldc}^{-/-}$larvae after applying a circular water current, as well as the time needed to immobilize in the dish (e.g., stabilize their position). While the siblings could actively swim against the water current in order to maintain their position, $\mathrm{gldc}^{-1-}$ mutants were unable to fight against it and to stabilize their position. As a result, they were passively carried through the dish by the water current in a lethargic and hypotonic fashion (Figure 3G). Consistently, the time to stabilize their position after applying water current was significantly increased compared with their siblings (Figure $3 \mathrm{H}$ ). Furthermore, we noticed an anomaly in the swimming balance of $\mathrm{gldc}^{-1-}$ larvae. Indeed, we observed that while the siblings were exploring their tank by swimming throughout it, most of the $\mathrm{gldc}^{-1-}$ larvae floated at the surface, unable to explore their environment, indicating a swimming balance impairment (Figure 3, C, I, and J). Of note is that although seizures are often reported in GE patients, electrophysiology recordings from larval brains did not detect any electrical discharge in $\mathrm{gldc}^{-/-}$fish (data not shown). Taken together, these results show that the $\mathrm{gldc}^{-1-}$ mutants displayed motor phenotypes reminiscent of GE symptoms in human patients, such as early lethality, hypotonia, and lethargy.

Metabolomics analysis indicates broad metabolic perturbations in gldc $^{-1}$ larvae. After characterizing the disease-related phenotype of $\mathrm{gldc}^{-1-}$ mutants, we sought to describe the molecular mechanisms involved in pathogenicity. Since glycine acts as an essential amino acid as well as a neurotransmitter in the CNS, we conducted a broad assay of metabolites by LC-MS in order to titrate the level of all proteinogenic and the main non-proteinogenic amino acids, as well as the main neurotransmitters. Interestingly, we found that extracts from $\mathrm{gldc}^{-1-}$ larvae showed a significant increase in many amino acids other than glycine, such as sarcosine, proline, valine, cystathionine, leucine, and arginine (Figure 4A). Moreover, we found that $\mathrm{gld}^{-1-}$ larvae showed a significant reduction in the levels of GABA and glutamate compared with $g l d c^{+/+}$ larvae (Figure 4B), whereas dopamine and serotonin levels were not significantly affected. Likewise, this suggests that $g l d c \mathrm{KO}$ impairs the metabolism of amino-acid/neurotransmitters other than glycine. We next sought to determine whether these changes were the result of an accumulation over time or if they were directly linked with GLDC KO. Thus, we performed the same LC-MS analysis at $2 \mathrm{dpf}$ and noticed that only sarcosine and glycine were already significantly increased in $\mathrm{gldc}^{-/}$embryos (Supplemental Figure 1; supplemental material available online with this article; https://doi.org/10.1172/jci.insight.124642DS1). This suggest that increases in proline, valine, cystathionine, leucine, and arginine levels occur over time. We also found that at this early stage, methionine was significantly decreased in mutant embryos, although this change did not persist until $7 \mathrm{dpf}$. 
A

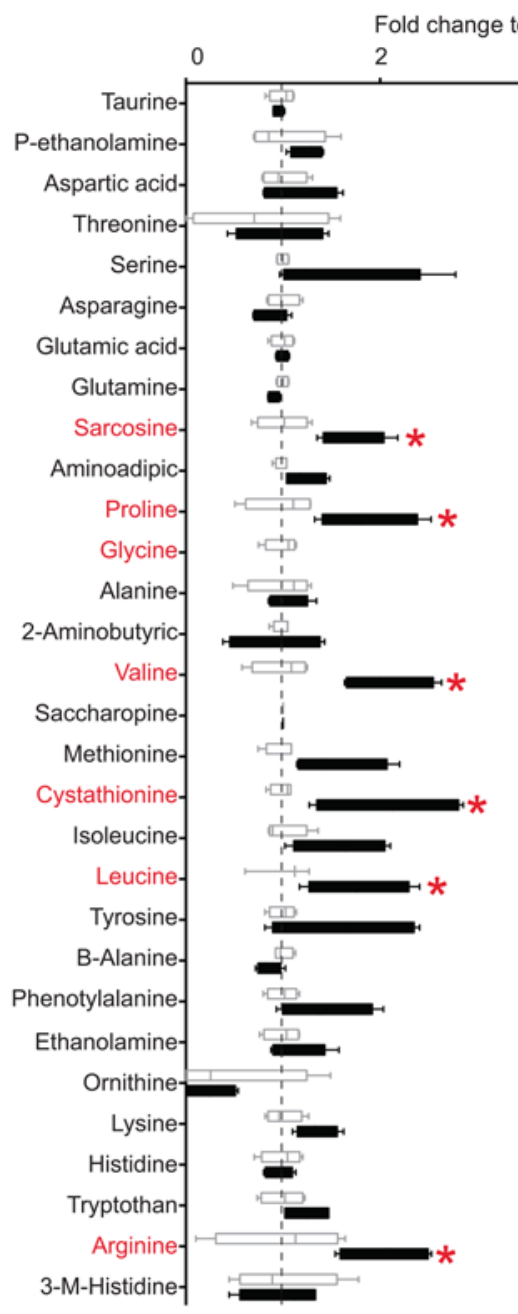

D

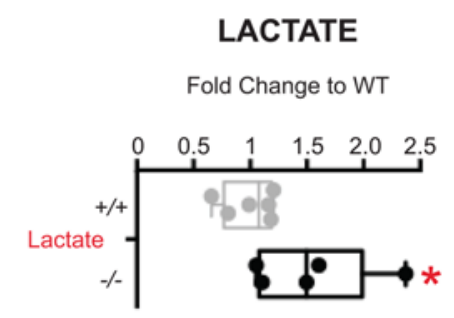

B

NEUROTRANSMITTERS

6

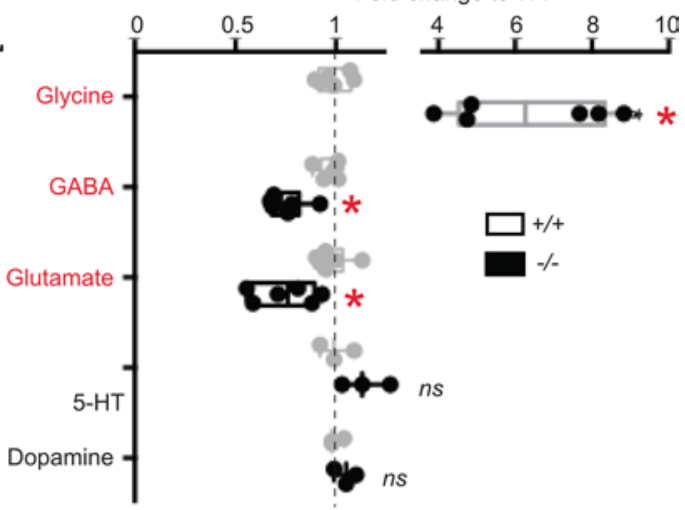

C FOLATE METABOLITES

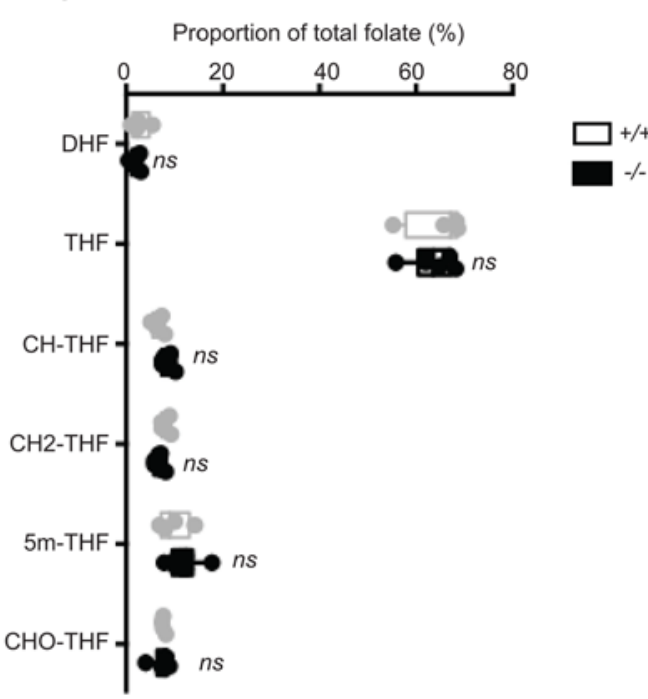

E

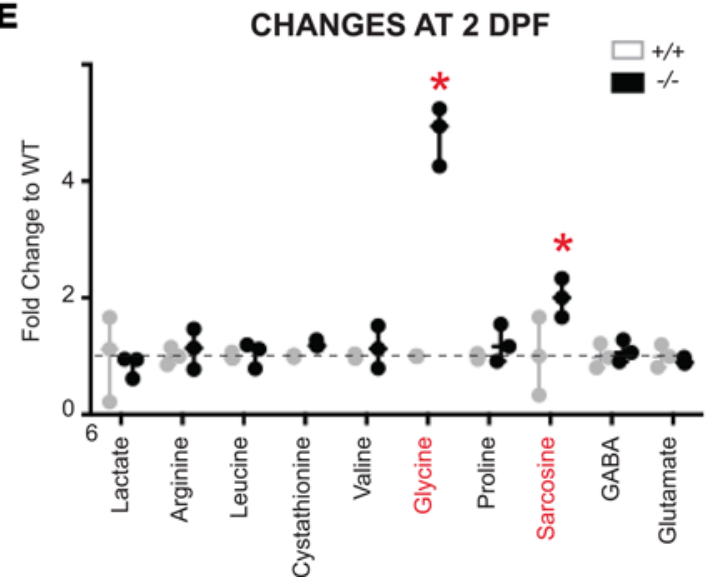

Figure 4. Metabolomics analysis indicates broad metabolic perturbations in gldc/- larvae. (A) LC-MS analysis of the levels of the main proteogenic and non-proteogenic amino acids at $7 \mathrm{dpf}$ reveals a significant increase in sarcosine, proline, glycine, valine, cystathionine, leucine, and arginine in gld $^{-/-}$compared with $g / d c^{+/+}$larvae ( $t$ test, respectively, $P=0.035, P=0.0062, P<0.00001, P=0.0006, P=0.0006, P=0.029$, and $P=0.0006$ ). (B)

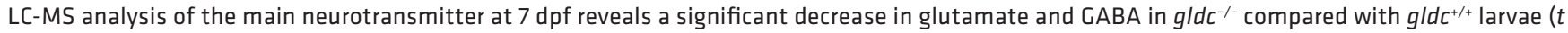
test, both $P<0.00001)$. Asterisks indicate significant differences. (C) LC-MS analysis of folate compounds revealed no difference between gld $^{-1 /-}$ and $\mathrm{gldc}^{+/+}$larvae at $7 \mathrm{dpf}$. Boxes and whiskers represent minimum and maximum values, and a line shows the median value. Each dot corresponds to an individual experiment $(N)$ with at least 3 fish $(n>3)$. DHF, dihydrofolate 5; me-THF, 5-methyl THF. (D) Lactate level titrages by LC-MS at 7 dpf shows an increase in $\mathrm{g} / \mathrm{dc} \mathrm{c}^{-/-}$larvae. (E) Same LC-MS analysis as in A, but in 2-dpf embryos. ${ }^{*} P<0.05$. 
The GCS is also involved in folate 1-carbon metabolism (FOCM), since by degrading glycine, gldc donates 1 carbon unit to this metabolic cycle $(7,15,16)$. Thus, we examined the levels of FOCM-related metabolites in $\mathrm{gldc}^{-/}$larvae in order to determine whether an imbalance in this metabolism is consistently observed in our fish model. However, LC-MS titration of different folate-related metabolites did not reveal any defect in FOCM in $\mathrm{gld}^{-1-}$ as compared with $g l d c^{+/+}$larvae (Figure $4 \mathrm{C}$ ). We also noted that tetrahydrofolate (THF) made up approximately $60 \%$ of total folate in zebrafish larvae. This was markedly different from mammalian (mouse and human) tissue or E. coli, in which 5-methyl THF or formyl-THF are abundant (15).

Finally, we also investigated the levels of lactate, since lactic acidosis is common in a number of metabolic inborn errors and has been reported in patients with GE (17-19). We found that the levels of lactate in $\mathrm{gldc}^{-1}$ mutants were significantly increased at $7 \mathrm{dpf}$. Interestingly, there was no difference in the levels of lactate in $\mathrm{gldc}^{-/}$and $\mathrm{gldc}^{+/+}$fish at $2 \mathrm{dpf}$, suggesting that there is a progressive accumulation of lactate that leads to lactic acidosis at $7 \mathrm{dpf}$, whereas the levels of glycine were already increased at $2 \mathrm{dpf}$ in $\mathrm{gldc}^{-1-}$ fish (Figure 4D).

Altogether, these results demonstrate that gldc loss of function induces previously unknown broad metabolic perturbations.

Transcriptomics analysis reveals differences in the expression of cell cycle-, proliferation-, and metabolism-related

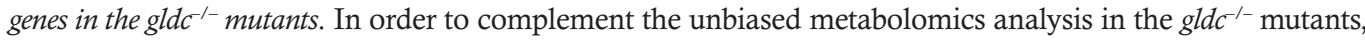
we carried out whole transcriptome analysis by deep sequencing of RNAs extracted from $7 \mathrm{dpf} \mathrm{gldc}^{-1-}$ and $\mathrm{gldc}^{+/+}$larvae. There were 408 differentially expressed genes in $\mathrm{gldc}^{-/-}$versus $\mathrm{gldc}^{+/+}$larvae, of which 153 were up- and 255 downregulated (Figure 5). Using gene clustering and pathway analysis from the list of significant differentially expressed genes, we identified multiple pathways whose gene expression was affected (Table 2). As expected, multiple genes involved in general metabolism are misregulated in $g l d c^{-1-}$ larvae. As an example, the amino acid transporter slc6a19 was downregulated in $\mathrm{gld}^{-1-}$ (fold change [FC] $-2.14, P=3.61 \times 10^{-16}$ ), and its mutation in human causes a metabolic disorder of neutral amino acid transport, Hartnup disease (Table 2 and ref. 20). Unexpectedly, only a few brain-specific genes were found to be differentially expressed. However, one of them, glycine transporter 1 (GlyT1; slc6a9), was downregulated (FC $\left.-1.61, P=1.65 \times 10^{-8}\right)$ in gld $^{-1}$ compared with $\mathrm{gldc}^{+/+}$fish, and is relevant in the context of a neurological disorder such as GE.

In addition, genes involved in cell adhesion and the extracellular matrix, such as integrin beta 4 (FC -1.5 , $\left.P=6.18 \times 10^{-5}\right)$ and laminin $3\left(\mathrm{FC}-1.70, P=1.29 \times 10^{-5}\right)$, were found to be downregulated in $\mathrm{gldc}^{-1-}$ mutants. Interestingly, many genes involved in the cell cycle (e.g., gadd45ba; FC 1.72, $P=3.39 \times 10^{-7}$ ), as well as known oncogenes (e.g., c-fos and mycb; FC: $1.90, P=1.89 \times 10^{-7}$, and FC 1.55, $P=1.03 \times 10^{-7}$ ) and tumor suppressor genes $\left(b c l 6 a\right.$; FC $\left.-1.45, P=1.03 \times 10^{-7}\right)$ were misregulated in $g l d c^{-1}$ compared with $\mathrm{gldc}^{+/+}$larvae.

Altogether, these transcriptomic data suggest that the majority of genes whose expression is affected by GLDC KO are related to metabolism and that, surprisingly, no major transcriptomic changes occur in the CNS. Moreover, these data indicated that cell-cycle homeostasis may be perturbed in $\mathrm{gldc}^{-1-}$ larvae, suggesting that cell proliferation may be affected in $\mathrm{gldc}^{-1-}$ mutants.

The main brain networks are not affected by gldc KO despite a transient decrease in proliferation in gldc $^{-1}$ brain. The imbalance in the expression of proliferation-related genes observed in $\mathrm{gld}^{-1-}$ mutants from the RNASeq analysis led us to investigate the proliferation homeostasis in the brain throughout neurodevelopment. Moreover, decreased proliferation at embryonic stages has been reported in a mouse model of GLDC KO (7). Thus, we performed IHC at different developmental time points (1, 3, and $7 \mathrm{dpf}$ ) using an antibody against the phosphorylated form of histone $3(\mathrm{pH} 3)$, which marks cells undergoing mitosis. Interestingly, this assay revealed a significant decrease in cellular proliferation in the brain of $\mathrm{gldc}^{-/-}$mutants at 1 and 3 dpf (Figure 6, A and I). However, this slight decrease in proliferation was not sustained until later stages, since no difference was observed at $7 \mathrm{dpf}$ between $\mathrm{gldc}^{-{ }_{-}}$and $\mathrm{gldc^{+/+ }}$ larvae (Figure 6I).

Since a difference in proliferation homeostasis at early stages of neurodevelopment may have an impact on the content of specific neural cell populations, we sought to investigate whether the major cell populations and/or structures of the brain neuronal networks were affected. First, we checked the general morphology of neuronal fibers in the brain of $\mathrm{gldc}^{-1-}$ larvae compared with their siblings by immunolabeling acetylated tubulin (ac-Tub). No difference in the labeling of axonal tracks in the brain of $\mathrm{gldc}^{-/-}$and $\mathrm{gldc}^{+/+}$larvae was observable at $24 \mathrm{hpf}$, nor at later stages (4 dpf) (Figure 6B). Then we aimed at checking the structure and neural content of (a) the GABAergic network using the [ $\mathrm{dlx} 5 / 6: \mathrm{G}$ FP] transgenic line (Figure 6C and ref. 21), (b) glutamatergic network using the [vglut:RFP] transgenic line (Figure 6D and ref. 22), (c) the dopamine network through immunolabeling against tyrosine hydroxylase $(\mathrm{TH})$ (Figure $6 \mathrm{E}$ ), as well as (d) the cerebellum through immunolabeling against paravalbumin 7 


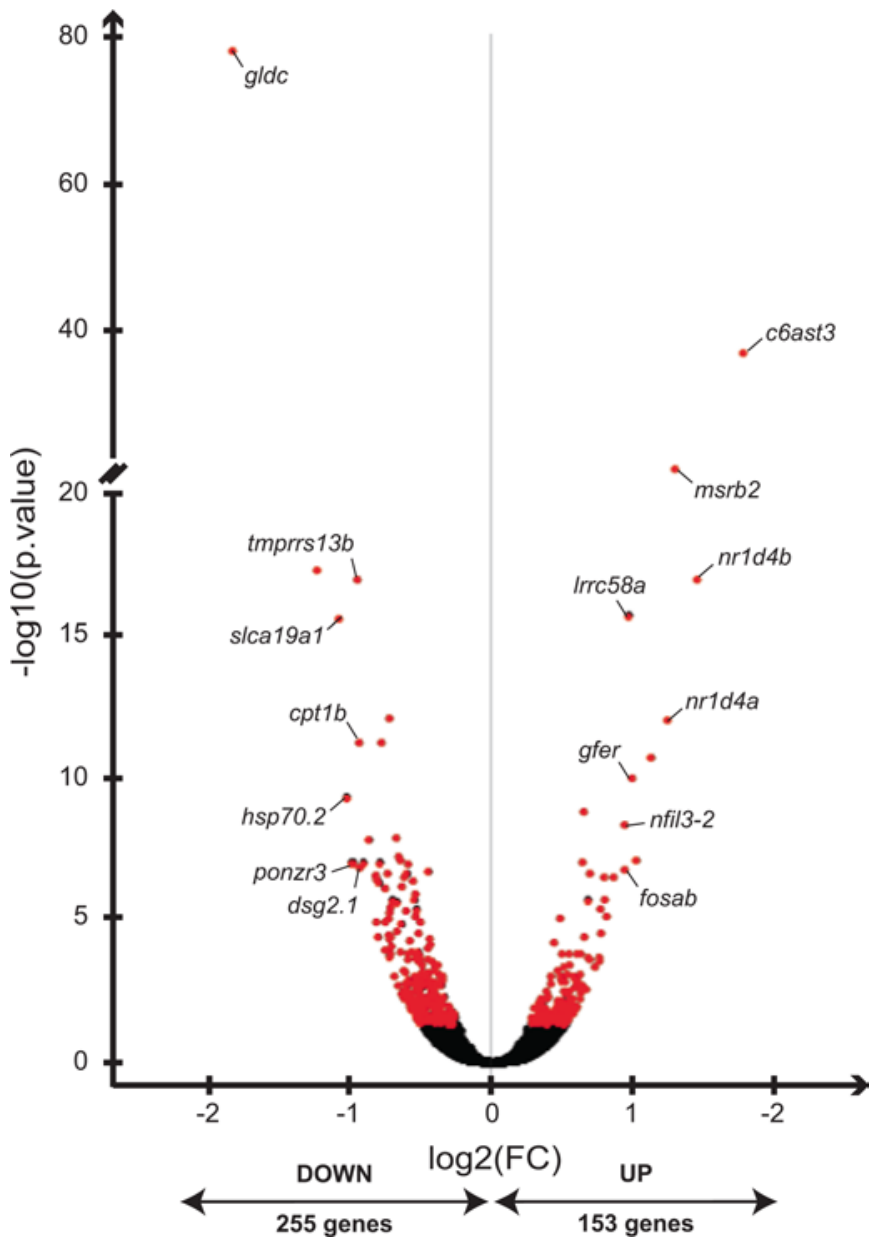

Figure 5. Transcriptomics analysis reveals differences in the expression of cell cycle-, proliferation-, and metabolism-related genes in the gldc $^{-1-}$ mutants. RNA sequencing analysis of 7-dpf larvae reveals that 408 genes are differentially expressed, with 255 upregulated and 153 downregulated, in gldc $c^{-1-}$ compared with gldc $c^{+/}$larvae.

(PAV7, labeling Purkinje cells) and vglut1 (labeling granule cells) (Figure 6, F and G). When comparing the cell content and/or immunolabeled morphology of these neuronal networks at different time points $(3,5$, and $7 \mathrm{dpf})$, we were unable to observe any differences in any of these brain network markers between $\mathrm{gldc}^{-1-}$ and $\mathrm{gldc^{+/+ }}$ brains. Finally, we also examined the branchiomotor neuron population using the [islet1:GFP] transgenic line, as it is a sensitive measure of neural tube abnormalities, and found no aberrant development or migration of trigeminal, facial, and vagal motor neurons in $\mathrm{gldc}^{-1-}$ mutants at $3 \mathrm{dpf}$ (Figure $6 \mathrm{H}$ and ref. 22). These results indicate that the transient proliferation deficit observed at 3 $\mathrm{dpf}$ in $\mathrm{gldc}^{-1-}$ mutants does not lead to any major morphological defect in other brain networks. Thus, it is likely to solely reflect a transient difference in the proliferating rate of neural progenitors that has no effect on brain neural content and therefore no specific relevance to the motor phenotype.

Genetically and pharmacologically counteracting the exacerbated hyperglycinemia at the synapse rescues the gld-1motor phenotype. Since our previous results suggest that GLDC KO does not affect neural networks, we hypothesized that the $\mathrm{gldc}^{-1-}$ phenotype may be mainly due to metabolic defects, as described above. As a neurotransmitter, glycine is involved in synaptic signaling through binding to glycine receptors at inhibitory synapses and to NMDA receptors at excitatory synapses. Thus, we wondered whether the hyperglycinemia observed in $\mathrm{gldc}^{-1}$ larvae was affecting both inhibitory and excitatory synaptic transmission. To test this, we performed a pharmacological assay treating $\mathrm{gldc}^{-/}$and control embryos with either a glycine receptor antagonist (strychnine) or an NMDA receptor antagonist (dextromethorphan). Overnight treatment with a low dose of strychnine, a dose not affecting WT larval behavior, significantly rescued the hypotonic swimming phenotype of $7 \mathrm{dpf} \mathrm{gldc}^{-1}$ larvae (Figure 7A). Moreover, acute treatment with dextromethorphan did not significantly rescue the early motor deficiency of $\mathrm{gldc}^{-/}$embryos at $21 \mathrm{hpf}$, but daily exposure rescued the swimming defect at $7 \mathrm{dpf}$ (Figure 7, B and C). These results show that the hyperglycinemia was indeed overactivating both glycine receptors and NMDA receptors throughout the brain, which contributed to the GE-related hypotonic motor phenotype. 
Table 2. Pathway analysis of the differentially regulated genes in the gldc ${ }^{-/-}$mutants

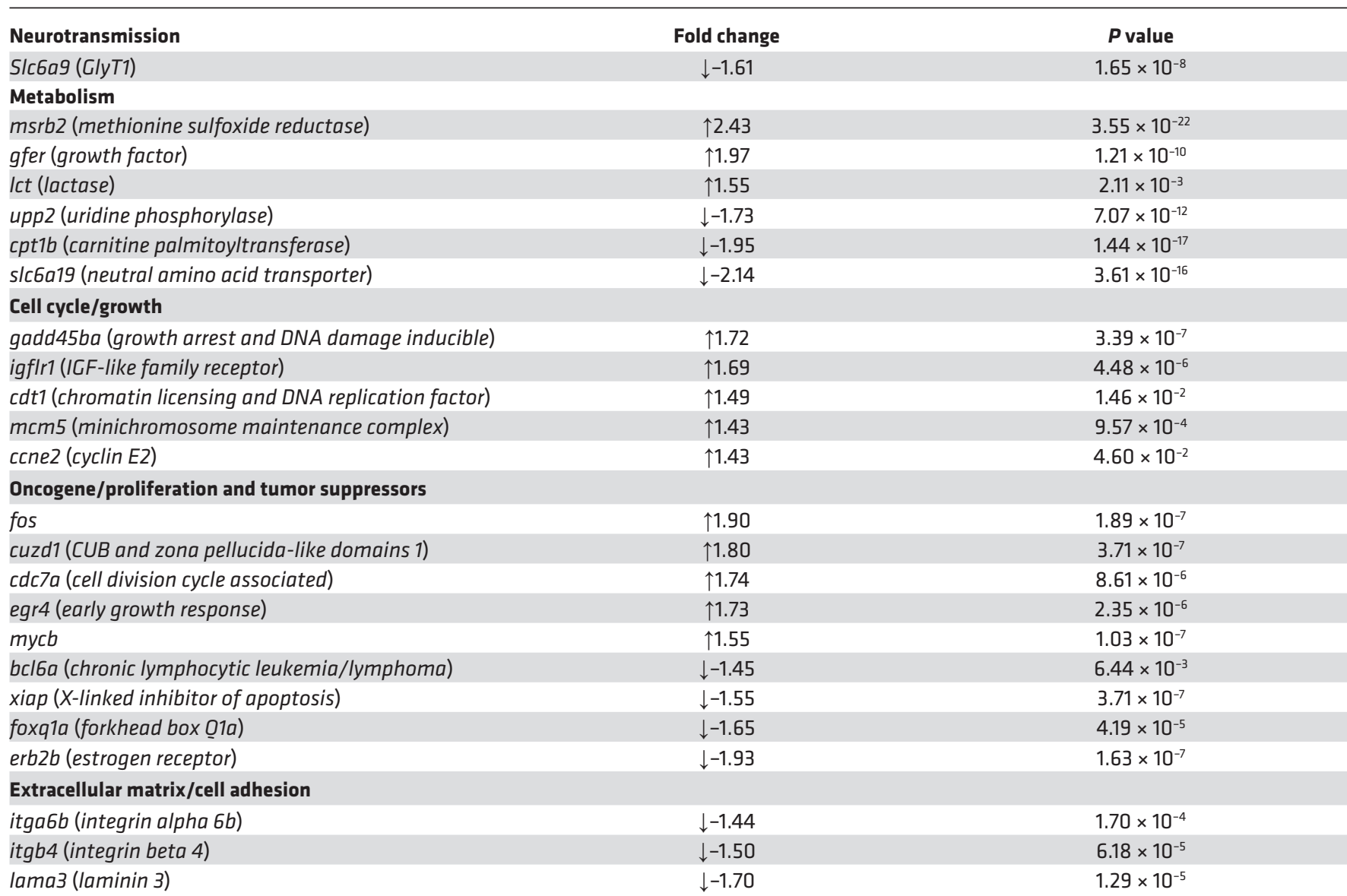

Pathway analysis reveals that genes involved in metabolism, cell cycle/growth, oncogene/proliferation and tumor suppressor, extracellular matrix, and one in neurotransmission are mis-regulated in $\mathrm{gld}^{-/-}$compared with $+/+$.

Then, we hypothesized that diminishing the hyperglycinemia at the synapse by overexpressing GlyT1 (a glycine transporter clearing glycine from the synaptic cleft) in $\mathrm{gldc}^{-/}$embryos may improve their motor deficits. To test this assumption, we cloned the GlyT1 cDNA and in vitro synthesized its mRNA in order to inject it in the first cell of $\mathrm{gldc}^{--}$or control embryos. We coinjected it with a GFP-encoding mRNA (as a positive readout) to allow us to select the GFP-positive embryo that overexpressed GlyT1 ubiquitously for up to $48 \mathrm{hpf}$. Interestingly, overexpression of GlyT1 in $\mathrm{gldc}^{-/-}$embryos was sufficient to completely rescue their early motor defect back to the level of their siblings (Figure 2D).

Altogether, these results confirm that the hyperglycinemia of $\mathrm{gldc}^{-1-}$ larvae affects normal glycinergic and NMDA signaling. Remarkably, counterbalancing this local hyperglycinemia at the synapse through the overexpression of GlyT1 completely rescued the motor phenotype of $\mathrm{gldc}^{-1}$ embryos (Figure 7E).

\section{Discussion}

In this work, we described 2 new cases of severe and mild GE and generated a zebrafish model of GE by knocking out gldc using CRISPR/Cas9, with the goal of characterizing the molecular mechanisms underlying GE. After showing the similarity of the $\mathrm{gldc}^{-1-}$ phenotype to the symptoms in severe GE patients, we sought unbiased changes in the metabolome and transcriptome, and our analysis revealed unexpected imbalances in the metabolism of amino acids and neurotransmitters other than glycine. Moreover, we found that $\mathrm{gld}^{-1}$ mutants display lactic acidosis preceding their death. They did not show obvious abnormalities in the folate profile. Given the cell type-specific expression of gldc at $7 \mathrm{dpf}$, it is possible that defect in 1-carbon supply is subtle in whole embryo samples. A key output of FOCM is nucleotide biosynthesis, and we found that cell proliferation in the brain was slowed during development, leading to a transient decrease in the 


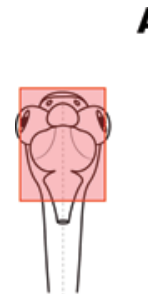

$\mathbf{A}$

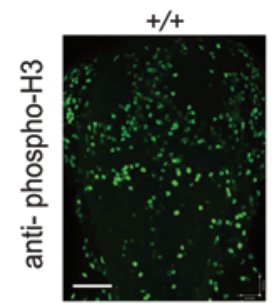

B

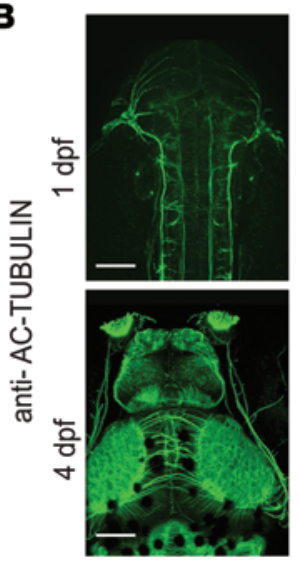

C

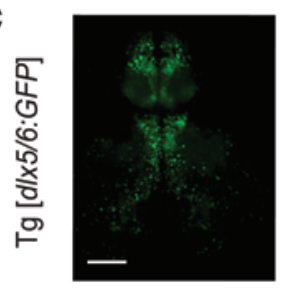

D

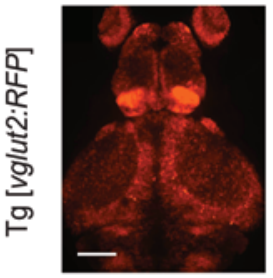

E

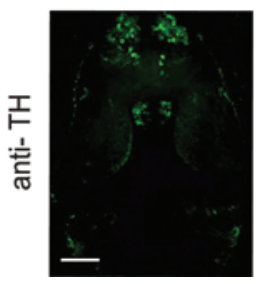

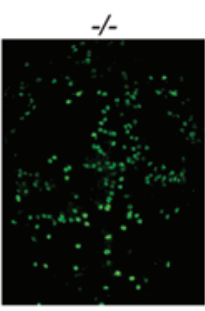
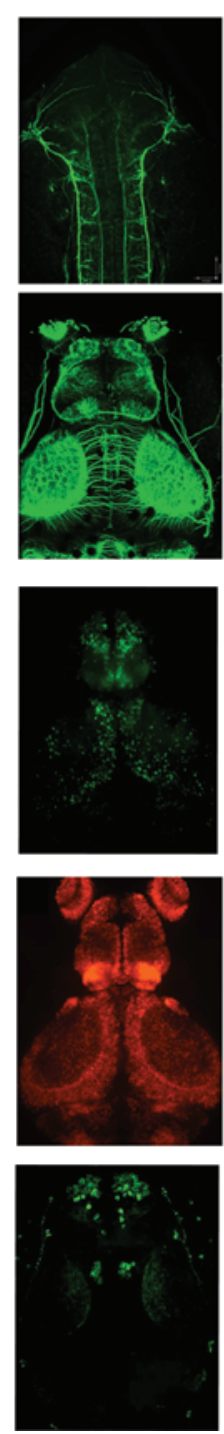

$\mathbf{F}$

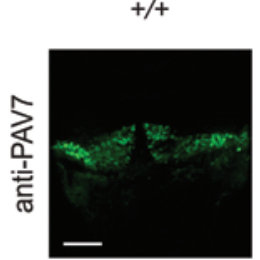

G

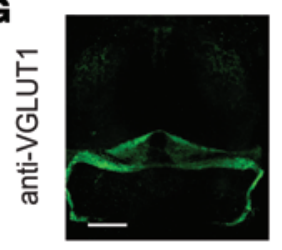

H
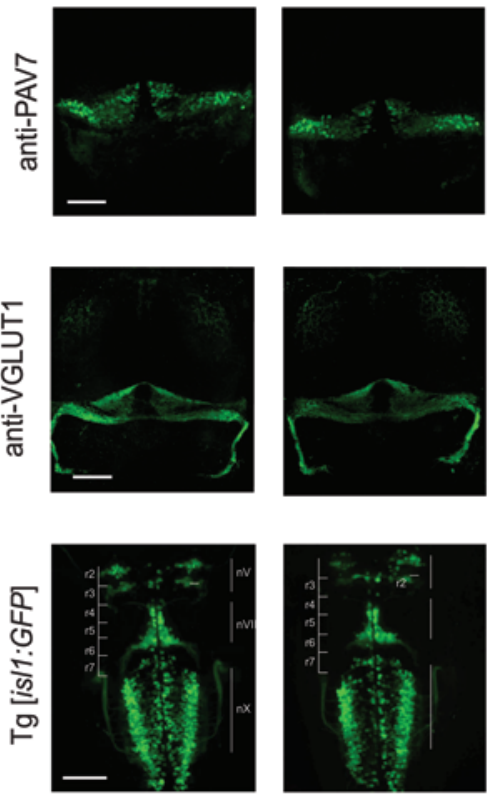

I
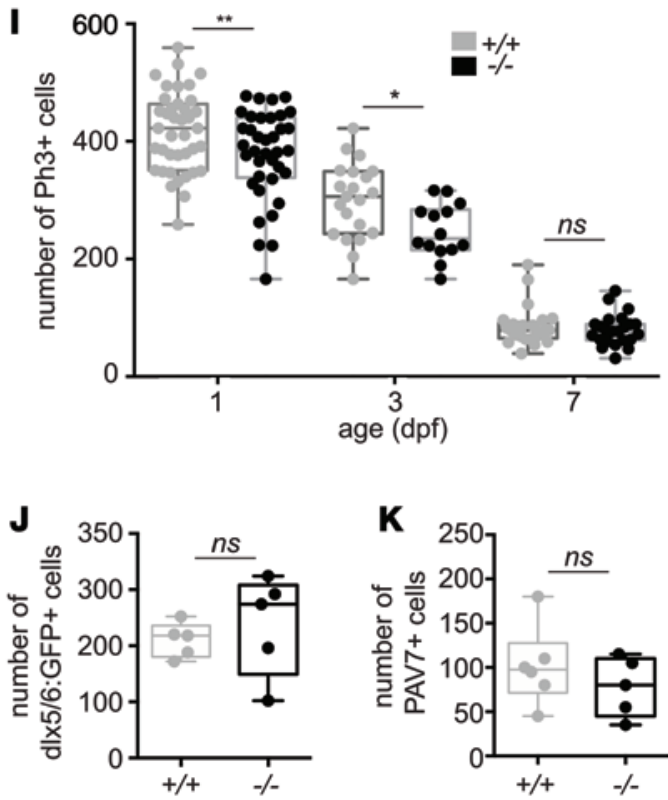

Figure 6. The main brain networks are not affected by gldc KO despite a transient decrease of proliferation in gldc ${ }^{-/-}$brain. (A) IHC against phospho-histone 3 (phospho-H3) labels proliferating cells in $\mathrm{g} / \mathrm{dc}^{-/-}$and $\mathrm{g} / \mathrm{dc} \mathrm{c}^{+/+} 3 \mathrm{dpf}$ larvae. (B) IHC against acetylated tubulin (AC-TUBULIN) labeling axonal tracks did not reveal any gross morphological difference at 1 and $4 \mathrm{dpf}$ between $\mathrm{gldc}^{-/-}$and $\mathrm{gldc^{+/+ }}$ larvae. (C) The transgenic dlx5/6:GFP line was used to visualize GABAergic cells in $\mathrm{gldc}^{-{ }^{--}}$and $\mathrm{g} / \mathrm{dc}^{+/+}$7-dpf larvae. (D) Using the vGluT2:RFP transgenic line to label the glutamatergic network did not reveal any gross morphological difference between $\mathrm{gld}^{-/-}$and $\mathrm{gldc^{+/+ }} 7$-dpf larvae. (E) IHC against tyrosine hydroxylase (TH) labeling dopaminergic neurons did not reveal any gross morphological difference between $\mathrm{gldc}^{-/-}$and $\mathrm{gldc}^{+/+}$at $5 \mathrm{dpf}$. (F) IHC against paravalbumin 7 (PAV7) labels Purkinje cells of the cerebellum on $5 \mathrm{dpf} g / d c^{-/-}$and $g / d c^{+/+}$larvae. (C) IHC against VGLUT1 labeling granule cells of the cerebellum did not reveal any gross morphological difference between 5-dpf $g / d c^{-/-}$and $g / d c^{+/+}$larvae. (H) Using the islet1:GFP transgenic line to label the branchiomotor neuron network did not reveal any gross morphological difference between $\mathrm{gldc}^{-/-}$and $\mathrm{g} / \mathrm{dc} \mathrm{c}^{+/+} \mathrm{3}$-dpf larvae. (I) Quantification of the number of phospho-H3positive $(\mathrm{Ph} 3+)$ cells revealed a significant decrease in proliferating cells in gld $c^{-/-}$compared with $g / d c^{+/+}$larvae at 1 and 3 dpf $\left(t\right.$ test, respectively, ${ }^{* *} P$

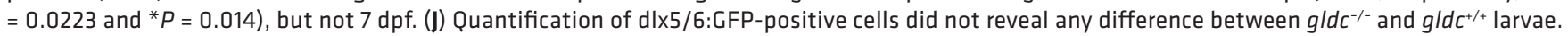
(K) Quantification of the number PAV7-positive cells did not reveal any difference between $\mathrm{gldc}^{-/-}$and $\mathrm{gldc}^{+/+}$. Boxes and whiskers represent minimum and maximum values, and a line shows the median value. Each dot corresponds to an individual biological replicate $(n>5)$. 
A
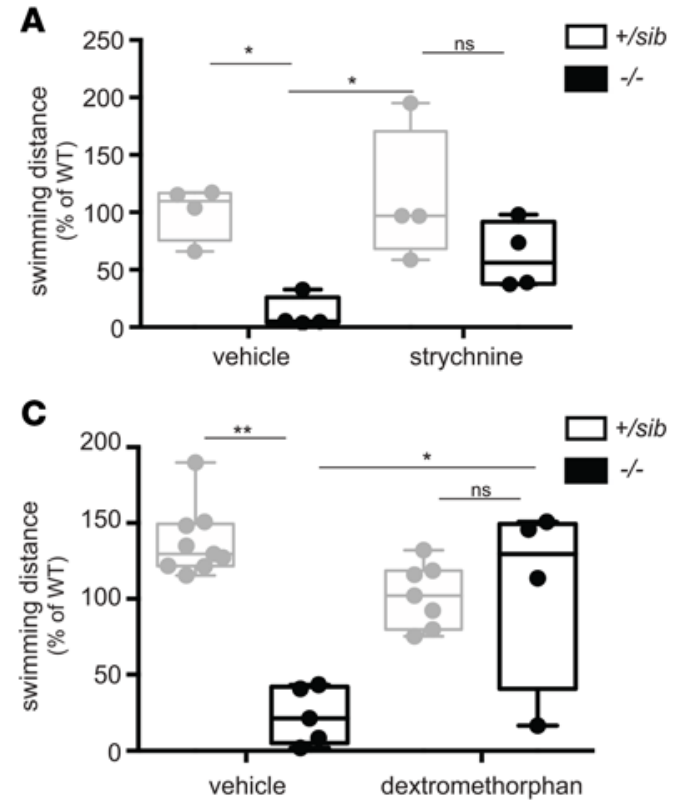

$\mathbf{E}$
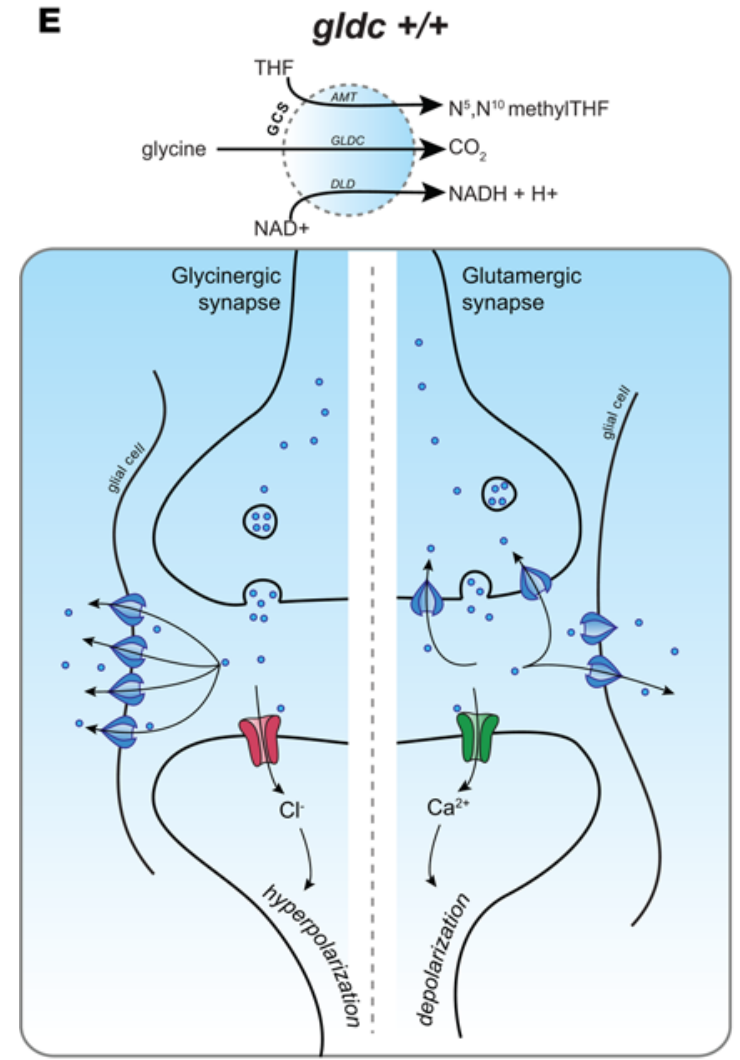

$\therefore$ glycine
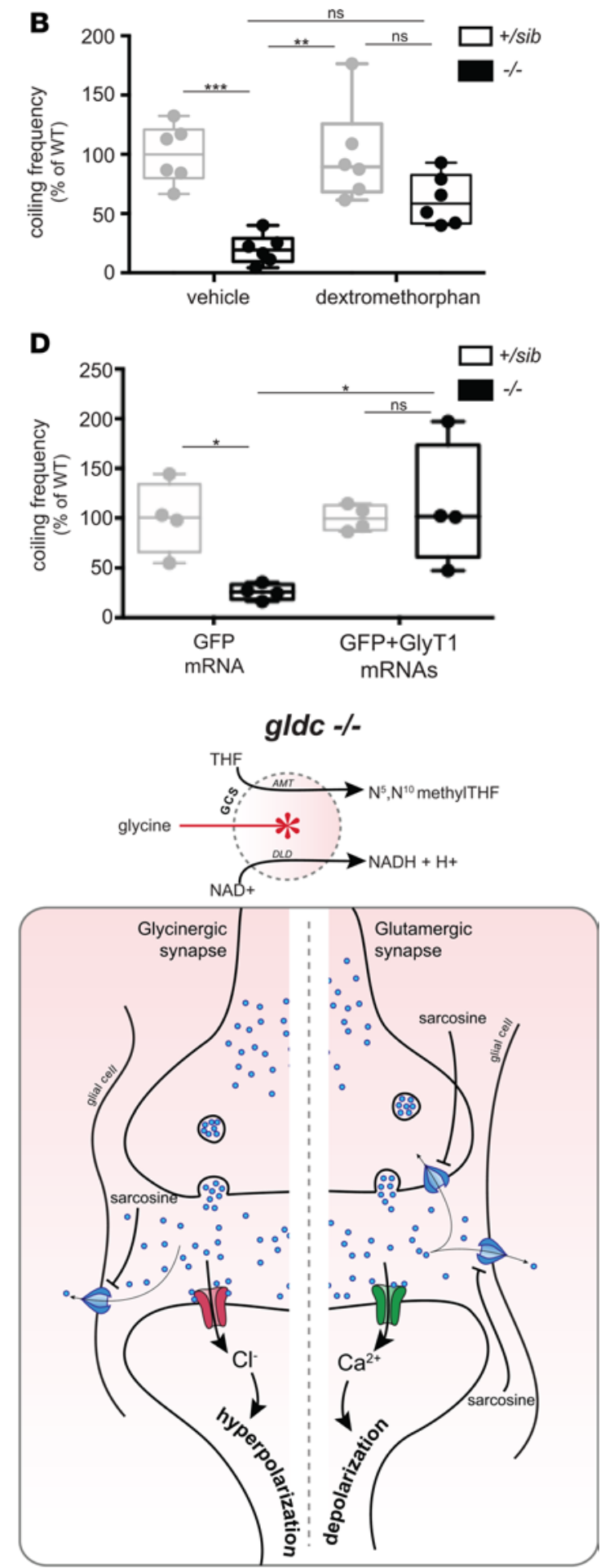

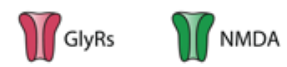

Figure 7. Genetically and pharmacologically counteracting the exacerbated hyperglycinemia at the synapse rescues the motor phenotype of gld $^{-/-}$larvae. (A) Overnight treatment with $5 \mu \mathrm{M}$ strychnine significantly rescues the hypotonic swimming of $g / d c^{-/-}$larvae $(n=6)$ at 7 days compared with vehicle-treated $g / d c^{-/-}$ $(n=11)$. (B) Overnight treatment with $50 \mu \mathrm{M}$ dextromethorphan decreases the hypotonic coiling of $\mathrm{gldc}^{-/-}(n=23)$ compared with vehicle-treated $\mathrm{gldc}^{-/-}$embryos $(n=27)$ at $21 \mathrm{hpf}$. (C) Daily $25-\mu \mathrm{M}$ dextromethorphan treatment over 7 days significantly rescues the hypotonic swimming of $\mathrm{gldc}^{-1-}(n=4)$ at 7 days, compared with vehicle-treated $g / d c^{-1-}$ larvae $(n=6)$. (D) First-cell stage glycine transporter 1 (GlyT1) mRNA injection rescues the coiling defect of $\mathrm{gld}^{-/-}$embryos at 21 hours after fertilization compared with GFP-injected $g / d c^{-/-}(n>15, N=4)$. Statistical analysis were performed with ANOVA, and significant results are shown with asterisks ( ${ }^{*} P<0.05 ;{ }^{* *} P<0.005 ;{ }^{* *} P<0.0001$ ). (E) Model representation of NMDA and glycine synapses of $\mathrm{gldc}^{-/-}$compared with $\mathrm{gldc^{+/+ }}$ fish. $\mathrm{gldc} \mathrm{c}^{-/-}$fish have exacerbated hyperglycinemia at these synapses due to a decrease in GlyT1 and an increase in the levels of sarcosine. Boxes and whiskers represent minimum and maximum values, and a line shows the median value. Each dot corresponds to an individual experiment $(N)$ of at least 5 fish $(n>5)$. 
number of dividing cells at 1 and $3 \mathrm{dpf}$. This is consistent with finding of diminished cell proliferation in the neuroepithelium of Gldc mutant embryos in mice (7). However, this was not associated with any gross morphological anomaly in the main brain structures or neuronal networks of $\mathrm{gldc}^{-1-}$ zebrafish, suggesting that this transient reduction of proliferation may be the consequence of metabolic perturbation, without having direct consequences for the motor phenotype. Finally, we confirmed that the hypotonic motor phenotype of $\mathrm{gldc}^{-1-}$ mutants is mainly due to the overactivation of NMDA and glycine receptors by glycine at the synapse. Interestingly, we showed that this hyperglycinemia at the synapse can be recovered by pharmacologically antagonizing these receptors, as well as by genetically increasing the expression of GlyT1.

Our findings suggest that there is exacerbated hyperglycinemia at the synapse of $\mathrm{gldc}^{-1-}$ mutants. Indeed, rather than compensating for the excess of glycine at the synapse, we found that some of the metabolic and transcriptomic changes induced by GLDC KO could worsen the level of glycine in the synaptic cleft. In fact, GlyT1 expression was significantly decreased in $g l d c^{-1-}$ mutants compared with their siblings, suggesting that the clearance of glycine from the cleft may be slowed. Moreover, our metabolomics analysis revealed a significant increase in sarcosine in $\mathrm{gldc}^{-/}$larvae, a non-proteogenic amino acid known to be a potent antagonist of GlyT1 activity. Indeed, sarcosine has raised significant therapeutic interest in recent years as a potential treatment for schizophrenia by increasing the glycinergic response in the brain of patients (23). Moreover, the level of sarcosine was already significantly elevated at $2 \mathrm{dpf}$ in $\mathrm{gldc}^{-1-}$ embryos, suggesting that this increase may be the result of a more direct effect of GLDC loss of function than the other changes in amino acid level that are accumulating over time. As a result, dampening the increase in the level of sarcosine from early stages may be an interesting experiment to test the relevance of targeting sarcosine therapeutically. Altogether, our findings highlight the fact that the elevated glycine level at the synapse may not be solely due to a defect of glycine degradation, but also to other synergic changes that may be good candidates as therapeutic targets (Figure 7E).

Consistent with the excess of glycine at the synapse, we showed that the overexpression of GlyT1 in the embryo was sufficient to fully rescue their motor condition. This is of prime interest for further therapeutic strategies targeting GlyT1 activity. Significant interest has arisen in developing potent antagonists of glycine transporters for the treatment of schizophrenia, increasing glycinergic synaptic signaling (23). In contrast, our results strongly suggest that an agonistic action on GlyT1 could be a key component for GE treatment. Here we propose that agonizing GlyT1 activity may have a broader action in the brain by counterbalancing hyperglycinemia at both the glycinergic and glutamatergic/NMDA synapse. However, the attenuation of glycine signaling in the brain can have important side effects that have to be considered, as exemplified by the poisoning effect of strychnine, a potent glycine receptor antagonist (24). As a result, such a therapeutic strategy would require the design and development of GlyT1 agonists with varying potency, whose activity could be efficiently adjusted to each GE patients.

At this juncture, our GLDC-KO zebrafish model could be of prime interest for drug screen purposes. Indeed, we showed that motor phenotypes relevant to the disease can be accurately observed and quantified as early as $20 \mathrm{hpf}$. Moreover, we used an automated quantification of coiling behavior that would allow for high-throughput phenotyping. As a result, our zebrafish line and our phenotyping assay could serve as an initial screening tool for testing the effects of newly designed drugs, such as GlyT1 agonists, that could then be tested in mammalian models. It is of note that many CNS-related disorders have been successfully modeled in the past, such as amyotrophic lateral sclerosis and spinal muscular atrophy, epilepsy, and autism (25-29).

Our work sheds light on another facet of GE, as our metabolomics analysis unraveled unexpected changes in metabolites other than glycine itself. Interestingly, some of the amino acids that are increased in gld $^{-1-}$ larvae (i.e., leucine and valine) are associated with another metabolic disease, maple syrup urine disease (MSUD). In patients with MSUD, the metabolism of branched-chain amino acids (BCAAs), such as leucine, isoleucine, and valine, is impaired due to mutations in subunits of the branched-chain $\alpha$-ketoacid dehydrogenase (BCKD). The levels of BCAA are thus increased in the urine, leading to a peculiar maple syrup smell. Remarkably, MSUD patients show some common symptoms to GE patients, such as hypotonia and seizures, and the only efficient treatment is a BCAA-free diet. In order to test the effect of an excess of valine or leucine on the motor phenotype of zebrafish, we treated WT embryos with these amino acids individually or in combination and found that they induced a reduction in coiling and swimming activity as strong as that when treated with glycine (data not shown). These results therefore suggest that valine and leucine may play a role in GE pathogenesis. However, GE patients' blood and urine samples are rarely tested for other amino acid levels, thus limiting our interpretation. 
We believe these findings should instigate more profound metabolic testing of human patients, such as dosage of BCAAs, lactate in the blood and/or urine, and glutamate and GABA in the CSF. This could allow identification of putative defects in metabolism of other amino acids and therefore open the opportunity for therapeutic strategies that may not have been considered, such as a BCAA-free diet. However, the fact that not all BCAAs were found to be elevated in $g l d c^{-1-}$ fish (such as isoleucine) suggests that this effect could not be generalized to all BCAAs but may rely on more specific mechanisms targeting leucine and valine. In this regard, a potential molecular link between GE and MSUD might involve the dihydrolipoamide dehydrogenase (DLD) subunit, which is part of both the GCS and the BCKD complexes. In humans, DLD deficiency leads to various symptoms, such as severe seizures and encephalopathy, muscle weakness, and accumulation of pyruvate, lactate, BCAAs, and the related metabolites $(30,31)$. Interestingly, we observed common phenotypes such as hypotonia, increased BCAAs (e.g., valine, leucine), as well as lactate in $\mathrm{gldc}^{-/-}$mutants, suggesting that $\mathrm{gldc} \mathrm{KO}$ might affect the activity of other metabolic complexes and therefore induce a broad metabolic defect. Our metabolomic assay also showed an increase in arginine in $\mathrm{gldc}^{-1-}$ larvae. It is worth noting that as a precursor of nitric oxide and polyamine, L-arginine regulates basic physiological functions, and it has been shown to be a player in age-related degenerative diseases such as Alzheimer's disease (32).

Previous studies by Greene et al. showed an imbalance in folate-related metabolites in Gldc-KO mice $(7,16)$. However, we did not detect any anomaly in the levels of FOCM compounds in gld $^{-1-}$ larvae (7). One possible explanation for this is that serine degradation, another source of 1-carbon units for the FOCM, could compensate for the loss of carbon units from glycine degradation (33), in addition, to the possibility that such an effect would be cell type- or stage-specific (see above). Moreover, the proportion of 1-carbon-carrying folates was found to be markedly lower in fish larvae than in mammalian tissue (pre- and postnatal). Thus, THF represents approximately $60 \%$ of total folate, perhaps suggesting a lesser requirement for 1-carbon-carrying folates in zebrafish larvae than in mammalian cells and tissues (mouse and human) or bacteria in which 5-methyl THF or formyl-THF is predominant (15). Thus, these results suggest that FOCM disturbances may not be directly involved in the motor dysfunction associated with GE, but rather specifically in structural malformations such as neural tube defects.

Altogether, the present work confirmed the central role played by hyperglycinemia in motor dysfunction associated with GE, but also unraveled an unexpected broader metabolic disturbance that could also be essential in GE pathogenesis. Both aspects of the disease should be considered in the design of new therapeutic strategies aimed at ensuring the survival of GE neonates as well as ameliorating surviving GE patients' quality of life. Among these strategies, our findings suggest that development of GlyT1 agonists, as well as a BCAA-free diet, would be a promising therapeutic avenue.

\section{Methods}

Genetic testing and glycine titration in patients. Blood samples from patients were tested for NKH by PCR amplification and direct DNA sequencing in both directions of the 9 exons of the $A M T$ gene and 25 exons of the GLDC gene, as well as the intron-exon borders. All genetic testing was performed at Denver Genetic Laboratories.

Fish husbandry and fish lines. WT zebrafish (Danio rerio) were reared at $28.5^{\circ} \mathrm{C}$, kept under a 12 -hour dark/12-hour light cycle, and staged as described previously (34). They were bred according to standard procedures (35). All experiments were performed in compliance with the guidelines of the Canadian Council for Animal Care and conducted at the Research Center of the University of Montreal Hospital Center (CRCHUM). The dlx5/6:GFP transgenic line was a gift from Marc Ekker (University of Ottawa), and the islet1:GFP and vglut2a:RFP transgenic lines were gifts from Shin-ichi Higashijima (Okazaki Institute for Integrative Bioscience) $(21,36)$.

Whole mount in situ hybridization and probe cloning. A specific gldc probe corresponding to the $5^{\prime}$ part of the coding sequence and first exon (951 bp amplified with the following primers: forward, GAAGGACCTTTGTGAGATTACGG; reverse, TAATGCAGGCCAGTGAGTGAG) was cloned within the pCS2+ vector using the TOPO TA cloning kit (Invitrogen). Whole-mount in situ hybridization of zebrafish embryos was performed as described in ref. 37.

Single-guide RNA and Cas 9 preparation and microinjection. The following gRNA sequence targeting the fourth exon of the gldc gene was designed using CRISPRscan (http://www.crisprscan.org/) (protospacer-adjacent motif [PAM] site is indicated in parentheses): GGGACACCTCGGGCTGGTA(CGG). Synthesis of gRNA 
and Cas9 mRNA was performed as described previously in ref. 12. WT embryos were collected for microinjection. A 1-nl drop of a mix of $100 \mathrm{ng} / \mu \mathrm{l}$ Cas $9 \mathrm{mRNA}$ and $30 \mathrm{ng} / 1 \mathrm{gRNA}$ was injected into 1-cell stage embryos.

$H R M$. Primers were designed using the Universal Probe Library Assay Design Center (Roche): forward, TTCAGTGAGTATTTGTGTTCTCTACAGG; reverse, TGGTCTGATAGTTGAGTAAGCTCTCC. The PCR reactions was performed as described in ref. 12.

Coiling analysis and swim tracking. 20-hpf embryos were embedded in low-melting-point agarose and covered with water; their movements inside the chorion were recorded using an iPhone 6S (Apple) camera fixed on a microscope adapter (ilabcam) for 20 minutes. DanioScope software (Noldus) was then used to quantify the number of bursts over time. At $7 \mathrm{dpf}$, larvae were transferred individually into a 96-well plate, and swim distance was recorded using Basler GenIcam camera and DanioVision recording chamber (Noldus). Analysis was performed using Ethovision XT 12 software (Noldus) to quantify the distance swam.

Hypotonia and swim balance tests. One gldc $^{-1-}$ mutant and one 7-dpf sibling larva were placed in the center of a water-filled petri dish, and a water current was manually applied. For the swimming balance test, a heat map tracking the movement of the larvae using Noldus DanioVision software was generated over 10 minutes.

LC-MS. Analysis of multiple folates was performed by ultra-performance LC-MS/MS as described previously (16). Briefly, folate sample buffer containing $20 \mathrm{mM}$ ammonia acetate, $0.1 \%$ ascorbic acid, $0.1 \%$ citric acid, and $100 \mathrm{mM}$ DTT at $\mathrm{pH} 7$ was added to the zebrafish samples. Each sample consisted of a pool of 3 zebrafish 6-7 days old. Samples were homogenized by sonication for 10 seconds using a handheld sonicator at $60 \% 1$ amplitude. Protein was removed by precipitation with addition of 2 sample volumes of acetonitrile, mixing for 2 minutes, and centrifugation for 15 minutes at $12,000 \mathrm{~g}$ and $4^{\circ} \mathrm{C}$. Supernatants were transferred to fresh tubes, lyophilized, and stored at $-80^{\circ} \mathrm{C}$ prior to analysis. Folates were measured by multiple reaction monitoring (MRM) with optimized cone voltage and collision energy for precursor and product ions. The folate distribution was calculated as relative amount of total folate in the sample and is expressed as percentage of the total folate. Amino acids were detected and quantified by LC-MS/MS as previously described $(38,39)$. Processing of the chromatograms obtained by LC-MS/MS was done using TargetLynx (Waters), including peak detection, peak integration, and concentration estimation based on calibration curves. For statistical analyses, Student's $t$ test was used. Significance testing and graphing was performed with GraphPad Prism 7 software. Analysis of the neurotransmitter and lactate was performed as described in ref. 40.

Transcriptomic assay, differential expression assay, and pathway analysis. Total RNA was extracted from 7-dpf $\mathrm{gldc}^{-/-}$and $\mathrm{gldc}^{+/+}$larvae using a PicoPure RNA extraction kit (Thermo Fisher Scientific) following the manufacturer's standard protocol. Quality of total RNA was assessed with BioAnalyzer Nano (Agilent), and all samples had an RNA integrity (RIN) number greater than 9.

Library preparation was done as described in ref. 41. Sequencing was performed with the Illumina NextSeq 500 using SBS Reagent Kit v3 (100 cycles, paired-end) with $1.6 \mathrm{nM}$ of the pooled library. Cluster density was targeted at around $800 \mathrm{k}$ clusters $/ \mathrm{mm}^{2}$. Between 73 and 98 million reads were generated for each sample. Library preparation and sequencing was done at the Institute for Research in Immunology and Cancer (IRIC) Platform (University of Montreal). About 95\% of high-quality reads were mapped onto the $\mathrm{Zv} 9$ version of the zebrafish genome (Ensembl release 77) using TopHat version 2.0.10.

Differential gene expression analysis was assessed by DESeq2 package using $\mathrm{R}$ software. Differential gene expression was filtered on an FDR (or adjusted $P$ value greater than 0.05 ). Pathway analysis was performed using DAVID bioinformatics resources (42). The list of differentially expressed genes was uploaded onto DAVID analysis wizard, and a list of all expressed genes found in our dataset was used as a background for gene enrichment analysis. The raw data have been deposited in the NCBI Sequence Read Archive (PRJNA490559).

IHC and pH3 and pav7 quantification. $\mathrm{gldc}^{-/-}$and $\mathrm{gldc}^{+/+}$embryos were anesthetized in $0.2 \%$ tricaine, fixed with $4 \%$ paraformaldehyde for 1.5 hours at room temperature (or in Dent's overnight at $4^{\circ} \mathrm{C}$ for $\mathrm{pH} 3 \mathrm{IHC}$ ). IHC was performed as previously described (43) with anti-ac-Tub (1:1,000, T6793, MilliporeSigma), antipH3 (polyclonal, 1:500, 06-570, Millipore), anti-TH (1:400, mab318, Millipore), anti-vGluT1 (1:500), or anti-pav7 (1:1,000, both gifts from Masahiko Hibi, Nagoya University, Nagoya, Japan). Quantification of Pav7- and pH3-positive cells was done blindly by using the count tool in Adobe Photoshop.

Drug treatments. All drugs were obtained through MilliporeSigma. The drug solutions were prepared as follows: $10 \mathrm{mM}$ stock solution of strychnine (S0532) was prepared by dissolving $33.4 \mathrm{mg}$ powder in $10 \mathrm{ml}$ water; $10 \mathrm{mM}$ stock solution of dextromethorphan (D9684) was prepared by dissolving $37 \mathrm{mg}$ powder in $10 \mathrm{ml}$ water. The stock solution was dissolved in fish water to reach the final concentration. Embryos and larvae were individualized in a glass beaker in a total volume of $50 \mathrm{ml}$ and were incubated overnight in a lightproof box at $28.5^{\circ} \mathrm{C}$. 
GlyT1 cloning and $m R N A$ in vitro synthesis. Full-length cDNA of GlyT1 was cloned from 24-hpf total RNAs using the following primers: forward, ATGAACAGCAGAAAGAATGGAGCA; reverse, CTATGCGCTGGGTGTGGG. The PCR product was cloned within the pCS2+ vector using TOPO TA cloning kit (Invitrogen). After sequencing, the corresponding mRNA was transcribed in vitro using SP6 RNA polymerase.

Statistics. Statistical analysis for comparing 2 groups was performed using a 2-tailed $t$ test (a $P$ value less than 0.05 was considered significant). When comparing more than 2 groups, the statistical analysis performed corrected for multiple comparisons and also for repeated measures when comparing multiple measurements between groups using Bonferroni's correction for multiple comparisons. Raw results are shown, with box and whiskers showing the minimum and maximum values as well as the median value (line).

Study approval. Genetic testing was performed under written consent from the patients. All experiments were performed in compliance with the guidelines of the Canadian Council for Animal Care and conducted at the Research Center of the University of Montreal Hospital Center (CRCHUM). Vertebrate animal welfare assurance from the IACUC for the use of adult zebrafish was approved on August 31, 2015.

\section{Author contributions}

$\mathrm{RR}$ and ES designed and performed all the zebrafish research experiments and wrote the manuscript. IAP, KYL, NL, and NDEG performed the MS analysis. ML performed the molecular biology research. KS and LAS performed the clinical study. PD, RR, and ES reviewed the manuscript.

\section{Acknowledgments}

We thank Marina Drits of the CRCHUM zebrafish platform, and Florent Guilloteau and Patrick Gendron of the IRIC genomic platform. This work was funded by the Grand Défi Pierre Lavoie, Savoy Foundation, Fonds de Recherche Québec Santé (FRQS), Rare Diseases Model and Mechanism Network, FRQS-affiliated Groupe de Recherche sur le Système Nerveux Central (GRSNC), Québec Ministère de l'Éducation, de l'Enseignement Supérieur et de Recherche (MEESR), and the CRCHUM. NDEG and KYL were funded by the Medical Research Council (N003713) and Joseph's Goal.

Address correspondence to: Éric Samarut; CRCHUM R09.706; 900 St. Denis Street; Montréal, Quebec H2X 0A9, Canada. Phone: 514.756.9429; Email: eric.samarut@umontreal.ca.

1. Applegarth DA, Toone JR. Glycine encephalopathy (nonketotic hyperglycinaemia): review and update. J Inherit Metab Dis. 2004;27(3):417-422.

2. Baker PR, et al. Variant non ketotic hyperglycinemia is caused by mutations in LIAS, BOLA3 and the novel gene GLRX5. Brain. 2014;137(Pt 2):366-379.

3. Korman SH, Gutman A. Pitfalls in the diagnosis of glycine encephalopathy (non-ketotic hyperglycinemia). Dev Med Child Neurol. 2002;44(10):712-720.

4. Hennermann JB, Berger JM, Grieben U, Scharer G, Van Hove JL. Prediction of long-term outcome in glycine encephalopathy: a clinical survey. J Inherit Metab Dis. 2012;35(2):253-261.

5. Coughlin CR 2nd, et al. The genetic basis of classic nonketotic hyperglycinemia due to mutations in GLDC and AMT. Genet Med. 2017;19(1):104-111.

6. Bjoraker KJ, et al. Neurodevelopmental outcome and treatment efficacy of benzoate and dextromethorphan in siblings with attenuated nonketotic hyperglycinemia. J Pediatr. 2016;170:234-239.

7. Pai YJ, et al. Glycine decarboxylase deficiency causes neural tube defects and features of non-ketotic hyperglycinemia in mice. Nat Commun. 2015;6:6388

8. Kojima-ishii K, et al. Model mice for mild-form glycine encephalopathy: behavioral and biochemical characterizations and efficacy of antagonists for the glycine binding site of N-methyl D-aspartate receptor. Pediatr Res. 2008;64(3):228-233.

9. Cui WW, et al. The zebrafish shocked gene encodes a glycine transporter and is essential for the function of early neural circuits in the CNS. J Neurosci. 2005;25(28):6610-6620

10. Swanson MA, Coughlin CR, Van Hove JL. Corrigendum: Swanson MA, Coughlin CR Jr, Scharer GH, et al: Biochemical and molecular predictors for prognosis in nonketotic hyperglycinemia. Ann Neurol 2015;78:606-618. Ann Neurol. 2016;79(3):505.

11. Swanson MA, et al. Biochemical and molecular predictors for prognosis in nonketotic hyperglycinemia. Ann Neurol. 2015;78(4):606-618

12. Samarut É, Lissouba A, Drapeau P. A simplified method for identifying early CRISPR-induced indels in zebrafish embryos using high resolution melting analysis. BMC Genomics. 2016;17:547.

13. Flusser H, Korman SH, Sato K, Matsubara Y, Galil A, Kure S. Mild glycine encephalopathy (NKH) in a large kindred due to a silent exonic GLDC splice mutation. Neurology. 2005;64(8):1426-1430.

14. Saint-Amant L, Drapeau P. Time course of the development of motor behaviors in the zebrafish embryo. $J$ Neurobiol. 1998;37(4):622-632.

15. Leung KY, De Castro SC, Cabreiro F, Gustavsson P, Copp AJ, Greene ND. Folate metabolite profiling of different cell types 
and embryos suggests variation in folate one-carbon metabolism, including developmental changes in human embryonic brain. Mol Cell Biochem. 2013;378(1-2):229-236.

16. Leung KY, et al. Partitioning of one-carbon units in folate and methionine metabolism is essential for neural tube closure. Cell Rep. 2017;21(7):1795-1808.

17. Applegarth DA, Toone JR. Nonketotic hyperglycinemia (glycine encephalopathy): laboratory diagnosis. Mol Genet Metab. 2001;74(1-2):139-146.

18. Hutchesson A, Preece MA, Gray G, Green A. Measurement of lactate in cerebrospinal fluid in investigation of inherited metabolic disease. Clin Chem. 1997;43(1):158-161.

19. Viola A, Chabrol B, Nicoli F, Confort-Gouny S, Viout P, Cozzone PJ. Magnetic resonance spectroscopy study of glycine pathways in nonketotic hyperglycinemia. Pediatr Res. 2002;52(2):292-300.

20. Kleta R, et al. Mutations in SLC6A19, encoding B0AT1, cause Hartnup disorder. Nat Genet. 2004;36(9):999-1002.

21. Yu M, Xi Y, Pollack J, Debiais-Thibaud M, Macdonald RB, Ekker M. Activity of dlx5a/dlx6a regulatory elements during zebrafish GABAergic neuron development. Int J Dev Neurosci. 2011;29(7):681-691.

22. Kimura Y, Hisano Y, Kawahara A, Higashijima S. Efficient generation of knock-in transgenic zebrafish carrying reporter/driver genes by CRISPR/Cas9-mediated genome engineering. Sci Rep. 2014;4:6545.

23. Tsai G, Lane HY, Yang P, Chong MY, Lange N. Glycine transporter I inhibitor, N-methylglycine (sarcosine), added to antipsychotics for the treatment of schizophrenia. Biol Psychiatry. 2004;55(5):452-456.

24. Boyd RE, Brennan PT, Deng JF, Rochester DF, Spyker DA. Strychnine poisoning. Recovery from profound lactic acidosis, hyperthermia, and rhabdomyolysis. Am J Med. 1983;74(3):507-512.

25. Schmid B, et al. Loss of ALS-associated TDP-43 in zebrafish causes muscle degeneration, vascular dysfunction, and reduced motor neuron axon outgrowth. Proc Natl Acad Sci U S A. 2013;110(13):4986-4991.

26. McGown A, et al. Early interneuron dysfunction in ALS: insights from a mutant sod1 zebrafish model. Ann Neurol. 2013;73(2):246-258

27. Oprea GE, et al. Plastin 3 is a protective modifier of autosomal recessive spinal muscular atrophy. Science. 2008;320(5875):524-527.

28. Baraban SC, Dinday MT, Hortopan GA. Drug screening in Scn1a zebrafish mutant identifies clemizole as a potential Dravet syndrome treatment. Nat Commun. 2013;4:2410.

29. Elsen GE, Choi LY, Prince VE, Ho RK. The autism susceptibility gene met regulates zebrafish cerebellar development and facial motor neuron migration. Dev Biol. 2009;335(1):78-92.

30. Quintana E, et al. Dihydrolipoamide dehydrogenase (DLD) deficiency in a Spanish patient with myopathic presentation due to a new mutation in the interface domain. J Inherit Metab Dis. 2010;33(supp1 3):S315-S319.

31. Brassier A, et al. Dihydrolipoamide dehydrogenase deficiency: a still overlooked cause of recurrent acute liver failure and Reyelike syndrome. Mol Genet Metab. 2013;109(1):28-32.

32. Yi J, Horky LL, Friedlich AL, Shi Y, Rogers JT, Huang X. L-arginine and Alzheimer's disease. Int J Clin Exp Pathol. 2009;2(3):211-238.

33. Locasale JW. Serine, glycine and one-carbon units: cancer metabolism in full circle. Nat Rev Cancer. 2013;13(8):572-583.

34. Kimmel CB, Ballard WW, Kimmel SR, Ullmann B, Schilling TF. Stages of embryonic development of the zebrafish. Dev Dyn. 1995;203(3):253-310

35. Westerfield M. The Zebrafish Book: A Guide for the Laboratory Use of Zebrafish (Danio rerio). 3rd ed. Eugene, Oregon, USA: Institute of Neuro Science; 1995.

36. Higashijima S, Hotta Y, Okamoto H. Visualization of cranial motor neurons in live transgenic zebrafish expressing green fluorescent protein under the control of the islet-1 promoter/enhancer. J Neurosci. 2000;20(1):206-218.

37. Thisse C, Thisse B. High-resolution in situ hybridization to whole-mount zebrafish embryos. Nat Protoc. 2008;3(1):59-69

38. Pena IA, et al. Pyridoxine-dependent epilepsy in zebrafish caused by Aldh7a1 deficiency. Genetics. 2017;207(4):1501-1518

39. Waterval WA, Scheijen JL, Ortmans-Ploemen MM, Habets-van der Poel CD, Bierau J. Quantitative UPLC-MS/MS analysis of underivatised amino acids in body fluids is a reliable tool for the diagnosis and follow-up of patients with inborn errors of metabolism. Clin Chim Acta. 2009;407(1-2):36-42.

40. Wojnicz A, Avendaño Ortiz J, Casas AI, Freitas AE, G López M, Ruiz-Nuño A. Simultaneous determination of 8 neurotransmitters and their metabolite levels in rat brain using liquid chromatography in tandem with mass spectrometry: application to the murine Nrf2 model of depression. Clin Chim Acta. 2016;453:174-181.

41. Swaminathan A, et al. Non-canonical mTOR-independent role of DEPDC5 in regulating GABAergic Network Development Curr Biol. 2018;28(12):1924-1937.e5.

42. Huang da W, Sherman BT, Lempicki RA. Systematic and integrative analysis of large gene lists using DAVID bioinformatics resources. Nat Protoc. 2009;4(1):44-57.

43. Puverel S, Nakatani H, Parras C, Soussi-Yanicostas N. Prokineticin receptor 2 expression identifies migrating neuroblasts and their subventricular zone transient-amplifying progenitors in adult mice. J Comp Neurol. 2009;512(2):232-242. 